\title{
Vertex Algebroids over Veronese Rings ${ }^{\star}$
}

\author{
Fyodor MALIKOV
}

Department of Mathematics, University of Southern California, Los Angeles, CA 90089, USA

E-mail: fmalikov@usc.edu

Received July 28, 2008, in final form December 07, 2008; Published online December 13, 2008

Original article is available at http://www.emis.de/journals/SIGMA/2008/086/

\begin{abstract}
We find a canonical quantization of Courant algebroids over Veronese rings. Part of our approach allows a semi-infinite cohomology interpretation, and the latter can be used to define sheaves of chiral differential operators on some homogeneous spaces including the space of pure spinors punctured at a point.
\end{abstract}

Key words: differential graded algebra; vertex algebra; algebroid

2000 Mathematics Subject Classification: 14Fxx, 81R10; 17B69

\section{Introduction}

Attached to a commutative associative algebra $A$ are the Lie algebra of its derivations, $\operatorname{Der}(A)$, and the module of Kähler differentials, $\Omega(A)$. The identities that are satisfied by the classic differential geometry operations, such as the Lie bracket, the Lie derivative, the de Rham differential, etc., can be summarized by saying that the $A$-module $\operatorname{Der}(A) \oplus \Omega(A)$ is a Courant algebroid, [8, 22]. For reasons that will become apparent later, we will use the notation $\mathcal{V}^{\text {poiss }}(A)=$ $\mathcal{V}^{\text {poiss }}(A)_{0} \oplus \mathcal{V}^{\text {poiss }}(A)_{1}$, where $\mathcal{V}^{\text {poiss }}(A)_{0}=A$ and $\mathcal{V}^{\text {poiss }}(A)_{1}=\operatorname{Der}(A) \oplus \Omega(A)$.

This example can be enriched in two different ways. First, it can be quantized. Attached to $A$ in [15] is the notion of a vertex algebroid, $\mathcal{V}(A)$. This notion is a result of axiomatizing the structure that is induced on conformal weight 0 and 1 components of a graded vertex algebra. One has $\mathcal{V}(A)=A \oplus \mathcal{V}(A)_{1}$ for some $\mathcal{V}(A)_{1}$, which fits in the exact sequence

$$
0 \rightarrow \Omega(A) \rightarrow \mathcal{V}(A)_{1} \rightarrow \operatorname{Der}(A) \rightarrow 0
$$

Therefore, $\mathcal{V}(A)_{1}$ is filtered and the corresponding graded object is $\operatorname{Gr} \mathcal{V}(A)_{1}=\mathcal{V}^{\text {poiss }}(A)_{1}$. This strongly resembles the Poincaré-Birkhoff-Witt filtration, and it is indeed true that the notion of a Courant algebroid is a quasiclassical limit of that of a vertex algebroid; this important observation is due to Bressler [7], but the fact that the Courant bracket belongs in the infinite dimensional world had been discovered by Dorfman much earlier, [9]. The relation of this notion to various string theory models has been elucidated in [23].

Unlike its quasiclassical counterpart, a vertex algebroid may not exist, and if it exists, it may not be unique. If $\operatorname{Spec}(A)$ is smooth, then [15] the obstruction to existence is the class $\operatorname{ch}_{2}(\operatorname{Spec}(A))$, and if $\operatorname{ch}_{2}(\operatorname{Spec}(A))=0$, then the isomorphism classes are parameterized by the hypercohomology group $H^{1}\left(\operatorname{Spec}(A), \Omega_{A}^{2} \stackrel{d_{D R}}{\rightarrow} \Omega_{A}^{3, \mathrm{cl}}\right)$. But what if $\operatorname{Spec}(A)$ is not $\operatorname{smooth}$ ?

This question suggests the second way to enrich, which is to note that $\operatorname{Der}(A)$ and $\Omega(A)$ tell the whole story only if $\operatorname{Spec}(A)$ is smooth; if not, then the higher algebras of derivations and modules of 1 -forms must arise. This was made precise by Hinich, [18], who defined $\operatorname{Der}(A)^{\bullet}$ as a kind of a derived functor of the functor Der by applying the latter to a polynomial differential

\footnotetext{
${ }^{\star}$ This paper is a contribution to the Special Issue on Kac-Moody Algebras and Applications. The full collection is available at http://www.emis.de/journals/SIGMA/Kac-Moody_algebras.html
} 
graded algebra resolution $R \rightarrow A$. This gives rise to a graded Courant algebroid functor $A \mapsto$ $\mathcal{V}^{\text {poiss }}(A)^{\bullet}$.

The problem of combining the two, that is to say, finding a quantization, $\mathcal{V}(A)^{\bullet}$, of $\mathcal{V}^{\text {poiss }}(A)^{\bullet}$ appears to be intellectually attractive and important for applications. If $A$ is a complete intersection, then the resolving algebra $R$ can be chosen to be a super-polynomial ring on finitely many generators, the corresponding resolution $R \rightarrow A$ being none other than the standard Koszul complex, and the quantization, a differential graded vertex algebroid $\mathcal{V}(R)$, is immediate; this observation has been used in a number of physics and mathematics papers. If, however, $A$ is not a complete intersection, then any resolving algebra $R$ is infinitely generated in which case defining a vertex algebroid $\mathcal{V}(R)$ becomes problematic because of various divergencies. A regularization procedure for some of these divergencies was suggested in [4] and elaborated on in [17].

Here is what we do in the present paper. Let $V_{N}$ be the $(N+1)$-dimensional irreducible $s l_{2^{-}}$ module, $O_{N} \subset \mathbb{P}\left(V_{N}\right)$ the highest weight vector orbit, and $A_{N}$ the corresponding homogeneous coordinate ring. All of this is a representation theorist's way of saying that $\mathbb{P}^{1} \stackrel{\sim}{\longrightarrow} O_{N} \subset \mathbb{P}^{N}$ is a Veronese curve, and $A_{N}$ is a Veronese ring.

$A_{N}$ is a quadratic algebra, in fact it is Koszul $[3,19,6]$, but it is not a complete intersection. The main result of the paper, Theorem 5.1.1, asserts that $\mathcal{V}^{\text {poiss }}\left(A_{N}\right)^{\bullet}$ admits a unique quantization. It is no surprise that this quantization, $\mathcal{V}\left(A_{N}\right)$, contains a vertex algebroid, $\mathcal{V}\left(s l_{2}\right)_{k}$, attached to $\widehat{s l}_{2}$ with some central charge $k$. What is more important is that the vertex algebroid attached to $\widehat{g l}_{2}$ enters the fray. The latter, $\mathcal{V}\left(g l_{2}\right)_{k_{1} k_{2}}$, depends in general on two central charges, $k_{1}, k_{2}$, and we find that the quantization conditions imply, first, that $k_{1}+k_{2}=-2$ and, second, that $k_{1}=-N-2$.

Theorem 5.1.1 and its proof appear in Section 5, and it is for the sake of this section that the paper was written. Section 4 is to a large extent an exposition of Hinich's result (see also [2]) with some extensions (Sections 4.3, 4.4, 4.5) that are needed in Section 5. Sections 2 and 3 are an attempt, perhaps futile, to make the paper self-contained - except Sections 3.7.3, 3.7.4, where sheaves of vertex algebroids over $\mathbb{C}^{2} \backslash 0$ are classified. The classification obtained is instrumental in proving Theorem 5.1.1; in particular, the vertex algebroid $\mathcal{V}\left(g l_{2}\right)_{k_{1}, k_{2}}$ with the compatibility condition $k_{1}+k_{2}=-2$ makes appearance in Section 3.7.4.

An obvious generalization of $A_{N}$ is provided by the homogeneous coordinate ring of a higher dimensional Veronese embedding $\mathbb{P}\left(\mathbb{C}^{n}\right) \rightarrow \mathbb{P}\left(S^{N}\left(\mathbb{C}^{n}\right)\right)$. We show (Theorem 5.3.1) that if $n>2$ and $N>1$, then no quantization exists.

Much of the above carries over to an arbitrary simple $\mathfrak{g}$, where $A_{N}$ is replaced with the homogeneous coordinate ring of the highest weigh vector orbit in the projectivization of a simple $\mathfrak{g}$-module. For example, $\mathbb{C}^{2} \backslash 0$ becomes the Bernstein-Gelfand-Gelfand base affine space, $G / N$. Constructed in [16] is the 1-parameter family of sheaves of vertex algebroids $H^{\infty / 2}\left(L \mathfrak{n}, \mathcal{V}_{G, k}\right)$ over $G / N$, where $\mathcal{V}_{G, k}, k \in \mathbb{C}$, is a family of vertex algebroids over $G,[1,10,14,16]$. There is little doubt that the family $H^{\infty / 2}\left(L \mathfrak{n}, \mathcal{V}_{G, k}\right), k \in \mathbb{C}$, is universal in that it classifies vertex algebroids over $G / N$ equipped with $\mathcal{V}(\mathfrak{g})_{k}$-structure. This is a higher rank analogue of the classification obtained in Section 3.7.3 and alluded to above. Note that just as $G / N$ is a $G \times T$ space, the maximal torus acting on the right, so there is a diagram of embeddings

$$
\mathcal{V}(\mathfrak{g})_{k_{1}} \hookrightarrow H^{\infty / 2}\left(L \mathfrak{n}, \mathcal{V}_{G, k}\right) \hookleftarrow \mathcal{V}(\mathfrak{t})_{k_{2}} \quad \text { with } \quad k_{1}+k_{2}=-\check{h}
$$

Therefore, $\mathcal{V}(\mathfrak{g})_{k} \oplus \mathcal{V}(\mathfrak{t})_{-k-h}$ is a higher rank analogue of $\mathcal{V}\left(g l_{2}\right)_{k,-k-2}$; here $\mathfrak{g}, \mathfrak{n}$ and $\mathfrak{t}$ are the Lie algebras of the Lie groups $G, N$, and $T$ resp. We elaborate on these remarks in Section 6 , where we use the technique of semi-infinite cohomology to compute CDO-s on some homogeneous spaces including the spaces of pure spinors punctured at a point. In the latter case, this gives an approach alternative to that of the original result by Nekrasov [24]. 
Some aspects of the $s l_{2}$-case, however, are not that easy to generalize. As they say, we hope to return to this subject in a separate paper.

We would like to conclude by saying that a major source of inspiration was provided to us by the work of Berkovits and Nekrasov [5, 4], where similar problems are analyzed in the case of the spinor representation of the spinor group.

\section{Vertex algebras}

2.1. Conventions. Underlying all the constructions in this paper will be the category of $\mathbb{Z}$ graded vector superspaces and grading preserving linear maps over $\mathbb{C}$. This grading will be called (and should be thought of as) the homological degree grading. More often, though, the attribute 'graded' will be skipped. Thus the phrase 'let $V$ be a vector space' will mean that $V=\oplus_{n \in \mathbb{Z}} V^{n}$, $V^{\text {even }}=\oplus_{n \in \mathbb{Z}} V^{2 n}, V^{\text {odd }}=\oplus_{n \in \mathbb{Z}} V^{2 n+1}$. Likewise, the prefix 'super-' will be usually omitted so that commutative will mean super-commutative, algebra super-algebra, bracket super-bracket: $[a, b]=a b-(-1)^{a b} b a$.

If $V$ and $W$ are vector spaces, then $V \otimes W$ is also a vector space with homological degree grading defined in the standard way so that $(V \otimes W)^{n}=\oplus_{i \in \mathbb{Z}} V^{i} \otimes W^{n-i}$. Various bilinear operations ('multiplications') to be used below will be morphisms of graded vector spaces $V \otimes$ $W \rightarrow U$.

Along with the homological degree grading, the grading by conformal weight will play a prominent role. The latter will be indicated by a subindex; thus, for example, the phrase 'a graded (by conformal weight) vertex algebra' will mean, in particular, a vector space $V$ with a direct sum decomposition $V=\oplus_{n \geq 0} V_{n}$ valid in the category of graded vector spaces.

Most of the definitions and constructions in this and the following section are well known, and their graded versions are always straightforward. We recommend [20] and [12] as an excellent introduction to vertex things and a guide to further reading.

Definition 2.2. A vertex algebra is a collection $\left(V, \mathbf{1}, T,{ }_{(n)}, n \in \mathbb{Z}\right)$, where $V$ is a vector space, $\mathbf{1} \in V$ is a distinguished element known as the vacuum vector, $T: V \rightarrow V$ is a linear operator known as the translation operator, each $(n)$ is a multiplication

$$
(n): \quad V \otimes V \rightarrow V, \quad a \otimes b \mapsto a_{(n)} b \quad \text { s.t. } \quad a_{(n)} b=0 \quad \text { if } \quad n \gg 0,
$$

that is subject to the following axioms:

(1) (vacuum)

$$
\mathbf{1}_{(n)}=\left\{\begin{array}{ll}
\operatorname{Id}_{V} & \text { if } n=-1, \\
0 & \text { otherwise, }
\end{array} \quad a_{(-1)} \mathbf{1}=a, \quad \forall a \in V ;\right.
$$

(2) (translation invariance)

$$
\left[T, a_{(n)}\right] b=(T a)_{(n)} b=-n a_{(n-1)} b, \quad \forall a, b \in V, \quad n \in \mathbb{Z} ;
$$

(3) (skew-symmetry)

$$
a_{(n)} b=(-1)^{a b} \sum_{j \geq 0}(-1)^{n+1+j} \frac{1}{j !} T^{j}\left(b_{(n+j)} a\right), \quad \forall a, b \in V, \quad n \in \mathbb{Z} ;
$$

(4) (Jacobi identity)

$$
\left[a_{(m)}, b_{(n)}\right] c=\sum_{j \geq 0}\left(\begin{array}{c}
m \\
j
\end{array}\right)\left(a_{(j)} b\right)_{(m+n-j)} c, \quad \forall a, b, c \in V, \quad m, n \in \mathbb{Z} ;
$$


(5) (quasi-associativity or normal ordering)

$$
\left(a_{(-1)} b\right)_{(n)} c=\sum_{j \geq 0} a_{(-1-j)} b_{(n+j)} c+(-1)^{a b} \sum_{j>0} b_{(n-j)} a_{(-1+j)} c, \quad \forall a, b, c \in V, \quad n \in \mathbb{Z} .
$$

The collection of axioms we used in Definition 2.2 is a little redundant but makes the exposition a little more transparent. It emphasizes the fact that the notion of a vertex algebra is a mixture of (appropriate analogues of) that of an associative algebra and a Lie algebra. Extracting the Lie part of the definition one arrives at the notion of a vertex Lie algebra.

Definition 2.3. A vertex Lie algebra is a collection $\left(V, T,_{(n)}, n \in \mathbb{Z}_{+}\right)$, where $V$ is a vector space, $T: V \rightarrow V$ is a linear operator, each ${ }_{(n)}$ is a multiplication

$$
{ }_{(n)}: \quad V \otimes V \rightarrow V, \quad a \otimes b \mapsto a_{(n)} b \quad \text { s.t. } \quad a_{(n)} b=0 \quad \text { if } \quad n \gg 0
$$

that is subject to the following axioms:

(1) translation invariance, that is, (2.2) for $n \geq 0$;

(2) skew-symmetry, that is, (2.3) for $n \geq 0$;

(3) Jacobi identity, that is, (2.4) for $n \geq 0$.

There is an obvious forgetful functor

$$
\Phi: \quad\{\text { Vertex algebras }\} \rightarrow\{\text { Vertex Lie algebras }\}
$$

Its left adjoint functor (the vertex enveloping algebra functor)

$$
\mathcal{U}: \quad\{\text { Vertex Lie algebras }\} \rightarrow\{\text { Vertex algebras }\}
$$

is well known to exist, see [25]; it also appears in [20] as 'the vertex algebra attached to a formal distribution Lie superalgebra'.

Note a canonical map

$$
\iota: \quad \mathcal{L} \rightarrow \Phi(\mathcal{U L})
$$

that is the image of $\operatorname{Id} \in \operatorname{Hom}(\mathcal{U L}, \mathcal{U L})$ under the identification $\operatorname{Hom}(\mathcal{U L}, \mathcal{U L}) \stackrel{\sim}{\longrightarrow} \operatorname{Hom}(\mathcal{L}, \Phi \mathcal{U L})$.

Example 2.4. Let $\mathcal{L}^{\prime}$ be a free $\mathbb{C}[T]$-module on one generator $L$ and let $\mathcal{L}(\mathcal{V} \text { ir })_{c}=\mathcal{L}^{\prime} \oplus \mathbb{C}$, where $\mathbb{C}$ is considered as a trivial $\mathbb{C}[T]$-module. $\mathcal{L}(\mathcal{V} \text { ir })_{c}$ carries a unique vertex Lie algebra structure such that

$$
L_{(0)} L=T(L), \quad L_{(1)} L=2 L, \quad L_{(2)} L=0, \quad L_{(3)} L=\frac{1}{2} c, \quad L_{(n)} L=0 \quad \text { if } n>3 .
$$

Upon quotienting out by the relation $\mathbf{1}=1$, the vertex enveloping algebra $\mathcal{U L}(\mathcal{V} \text { ir })_{c}$ becomes the vacuum representation of the Virasoro algebra of central charge $c$.

Example 2.5. Let $\mathfrak{g}$ be a Lie algebra with an invariant bilinear form $(\cdot, \cdot)$. Let

$$
\mathcal{L}(\mathfrak{g})_{k}=\mathbb{C}[T] \otimes \mathfrak{g} \oplus \mathbb{C} .
$$

This space carries an obvious action of $T$, where again we consider $\mathbb{C}$ as a trivial $\mathbb{C}[T]$-module, and a unique vertex Lie algebra structure such that

$$
(1 \otimes a)_{(0)}(1 \otimes b)=1 \otimes[a, b], \quad(1 \otimes a)_{(1)}(1 \otimes b)=k(a, b) .
$$


Upon quotienting out by the relation $\mathbf{1}=1$, the vertex enveloping algebra $\mathcal{U L}(\mathfrak{g})_{k}$ becomes the vacuum representation of the corresponding affine Lie algebra of central charge $k$.

If $\mathfrak{g}$ is chosen to be $g l_{N}=s l_{N} \oplus \mathbb{C} \cdot I$, then this construction has the following version: we let $(a, b)=\operatorname{tr}(a \cdot b), \mathcal{L}\left(g l_{N}\right)_{k_{1}, k_{2}}=\mathcal{L}\left(s l_{N}\right)_{k_{1}} \oplus \mathbb{C}[t] \otimes \mathbb{C} \cdot I$ and extend $(2.10)$ by

$$
(1 \otimes I)_{(1)}(1 \otimes I)=k_{2} N, \quad(1 \otimes I)_{(0)}(1 \otimes I)=(1 \otimes I)_{(n)}\left(1 \otimes s l_{N}\right)=0 \quad \forall n .
$$

In order to handle the case of the trivial bilinear form $(\cdot, \cdot)$, or more generally the case where $(\cdot, \cdot)$ is not unique even up to proportionality, we will change the notation and denote by $\mathcal{L}(\mathfrak{g})_{(\cdot, \cdot)}$ the vertex Lie algebra which is precisely $\mathcal{L}(\mathfrak{g})_{k}$ except that the last of conditions (2.10) is replaced with

$$
(1 \otimes a)_{(1)}(1 \otimes b)=(a, b)
$$

for some $(\cdot, \cdot)$.

A passage to the quasiclassical limit is a gentler way to blend the Lie and commutative/associative algebra parts of the structure.

Definition 2.6. A vertex Poisson algebra is a collection $\left(V, \mathbf{1} \in V, T,{ }_{(n)}, n \geq-1\right)$, where $V$ is a vector space, $T: V \rightarrow V$ is a linear operator, each ${ }_{(n)}$ is a multiplication

$$
(n): \quad V \otimes V \rightarrow V, \quad a \otimes b \mapsto a_{(n)} b \quad \text { s.t. } \quad a_{(n)} b=0 \quad \text { if } \quad n \gg 0
$$

that is subject to the following axioms:

(1) the triple $(V, \mathbf{1}, T,(-1))$ is a unital commutative associative algebra with derivation;

(2) the collection $\left(V, \mathbf{1} \in V, T,{ }_{(n)}, n \geq 0\right)$ is a vertex Lie algebra;

(3) each multiplication ${ }_{(n)}, n \geq 0$, is a derivation of $(-1)$.

Vertex Poisson algebras are to vertex algebras what Poisson algebras are to noncommutative algebras. The following construction (cf. [21]) is meant to illustrate this point.

2.7. Vertex algebras with filtration. Suppose a vertex algebra $V$ carries an exhaustive increasing filtration by vector spaces

$$
\mathbb{C} 1 \in F^{0} V \subset F^{1} V \subset \cdots \subset F^{p} V \subset \cdots, \quad \cup_{p \geq 0} F^{n} V=V
$$

which satisfies $\left(F^{p} V\right)_{(n)} F^{q} V \subset F^{p+q} V$ and $T\left(F^{p} V\right)=F^{p} V$ for all $p, q \in \mathbb{Z}$ so that $\left(F^{p} V\right)_{(n)} F^{q} V$ $\subset F^{p+q-1} V$ if $n \geq 0$. By focusing on symbols one discovers that the vertex algebra structure on $V$ defines the following on the corresponding graded object $\operatorname{Gr} V=\oplus_{p} F^{p} V / F^{p-1} V$ :

$$
\begin{aligned}
\mathbf{1}^{\mathrm{gr}}=\mathbf{1} \in F^{0} V ; & \\
T^{\mathrm{gr}}: & F^{p} V / F^{p-1} V \rightarrow F^{p} V / F^{p-1} V, \quad T(\bar{a})=T(a) \quad \bmod F^{p-1} V ; \\
(-1)^{\mathrm{gr}}: & \left(F^{p} V / F^{p-1} V\right) \otimes\left(F^{q} V / F^{q-1} V\right) \rightarrow F^{p+q} V / F^{p+q-1} V, \\
& \quad \bar{a} \otimes \bar{b} \mapsto \bar{a}_{(-1)^{g r}} \bar{b}=a_{(-1)} b \quad \bmod F^{p+q-1} V ; \\
(n)^{\mathrm{gr}}: \quad & \left(F^{p} V / F^{p-1} V\right) \otimes\left(F^{q} V / F^{q-1} V\right) \rightarrow F^{p+q-1} V / F^{p+q-2} V, \\
& \bar{a} \otimes \bar{b} \mapsto \bar{a}_{(n)^{g r}} \bar{b}=a_{(n)} b \quad \bmod F^{p+q-2} V \quad \text { if } \quad n \geq 0 .
\end{aligned}
$$

It is then immediate to check that $\left(\mathrm{Gr} V, \mathbf{1}^{\mathrm{gr}}, T^{\mathrm{gr}},{ }_{(n)} \mathrm{gr}^{\mathrm{g}}, n \geq-1\right)$ is a vertex Poisson algebra. For example, commutativity of the product ${ }_{(-1)}$ follows from the $n=-1$ case of (2.3) and associativity from the $n=-1$ case of (2.5).

The vertex algebras reviewed in Examples 2.4, 2.5 possess a filtration with the indicated properties - as does any vertex enveloping algebra: 
- in the case of $\mathcal{U L}(\mathcal{V} \text { ir })_{c}$ the filtration is determined by assigning degree one to $\iota\left(\mathcal{L}(\mathcal{V} \text { ir })_{c}\right)$, see $(2.9)$;

- in the case of $\mathcal{U L}(\mathfrak{g})_{k}$ the filtration is determined by assigning degree one to $\iota\left(\mathcal{L}(\mathfrak{g})_{k}\right)$.

Denote thus defined vertex Poisson algebras as follows:

$$
\begin{aligned}
& \mathcal{U}^{\text {poiss }} \mathcal{L}(\mathcal{V} \text { ir })=\operatorname{Gr} \mathcal{U} \mathcal{L}(\mathcal{V} \text { ir })_{c}, \quad \mathcal{U}^{\text {poiss }} \mathcal{L}(\mathfrak{g})=\operatorname{Gr} \mathcal{U} \mathcal{L}(\mathfrak{g})_{k}, \\
& \mathcal{U}^{\text {poiss }} \mathcal{L}\left(g l_{N}\right)=\operatorname{Gr} \mathcal{U} \mathcal{L}\left(g l_{N}\right)_{k, k_{2}} .
\end{aligned}
$$

\section{Courant and vertex algebroids}

Definition 3.1. A vertex (vertex Poisson) algebra $V$ is called graded if

$$
\begin{aligned}
& V=\bigoplus_{n=-\infty}^{+\infty} V_{n} \quad \text { so that } \\
& V_{n}=0, \quad \text { if } n<0, \quad \mathbf{1} \in V_{0}, \quad T\left(V_{m}\right) \subset V_{m+1}, \quad \text { and } \quad\left(V_{m}\right)_{(j)} V_{n} \subset V_{m+n-j-1} .
\end{aligned}
$$

Such grading is usually referred to as conformal, $V_{n}$ is called the conformal weight $n$ component, and $v \in V_{n}$ is said to have conformal weight $n$; the conformal weight of $v \in V_{n}$ is usually denoted by $\Delta(v)$.

All the vertex algebras we have seen are graded: $\mathcal{U L}(\mathcal{V} \text { ir })_{c}$ is graded by letting $V_{0}=\mathbb{C}$, $V_{1}=\{0\}, V_{2}=\iota(L), \mathcal{U L}(\mathfrak{g})_{k}$ by letting $V_{0}=\mathbb{C}, V_{1}=\iota(\mathfrak{g}), \mathcal{U L}\left(g l_{N}\right)_{k_{1}, k_{2}}$ by letting $V_{1}=\iota\left(g l_{N}\right)$

A graded vertex (vertex Poisson) algebra structure on $V$ induces the following structure on the subspace $V_{0} \oplus V_{1}$ :

$$
\begin{aligned}
& \mathbf{1} \in V_{0}, \\
& T: \quad V_{0} \rightarrow V_{1}, \\
& (n): \quad V_{i} \otimes V_{j} \rightarrow V_{(i+j-n-1)}, \quad n \geq 0, \quad i, j=0,1, \\
& (-1): \quad\left(V_{0} \otimes V_{i}\right) \oplus\left(V_{i} \otimes V_{0}\right) \rightarrow V_{i}, \quad i=0,1 .
\end{aligned}
$$

These data satisfy a list of identities obtained by inspecting those listed in Definitions 2.2 and 2.6 and choosing the ones that make sense. The meaning of 'make sense' is clear in the case of identities, such as the Jacobi, involving only operations $(n)$ with $n \geq 0$, because $V_{0} \oplus V_{1}$ is closed under these operations. Expressions $T^{j}\left(a_{(n)} b\right), a_{(i)} b_{(j)} c,\left(a_{(i)} b\right)_{(j)} c, a, b, c \in V_{0}, V_{1}$, are said to make sense if either they are compositions of operations (3.2)-(3.5) or because $\Delta(a)+\Delta(b)-n-1<0, \Delta(b)+\Delta(c)-j-1<0, \Delta(a)+\Delta(b)-i-1<0$ (resp.) in which case the expressions are defined to be 0 , cf. condition (3.1). Finally, we shall say that an identity makes sense if all the expressions that it involves make sense.

Definition 3.2. A Courant algebroid is a vector space $V_{0} \oplus V_{1}$ carrying the data (3.2)-(3.5) so that all those axioms of Definition 2.6 that make sense are valid.

Definition 3.3. A vertex algebroid is a vector space $V_{0} \oplus V_{1}$ carrying the data (3.2)-(3.5) so that all those axioms of Definition 2.2 that make sense are valid.

There are two obvious forgetful functors

$$
\begin{aligned}
& \Phi_{\text {alg }}: \quad\{\text { Vertex algebras }\} \rightarrow\{\text { Vertex algebroids }\}, \\
& \Phi_{\text {alg }}^{\text {poiss }}: \quad\{\text { Vertex Poisson algebras }\} \rightarrow\{\text { Courant algebroids }\},
\end{aligned}
$$

and both afford the left adjoints $\mathcal{U}_{\text {alg }}$ and $\mathcal{U}_{\text {alg }}^{\text {poiss }}$, which are analogous to (2.8). 
When applied to the algebras of (2.14) and of Examples 2.4, 2.5, they give us first examples of Courant

$$
\mathcal{V}^{\text {poiss }}(\mathcal{V} \text { ir })=\mathbb{C}, \quad \mathcal{V}^{\text {poiss }}(\mathfrak{g})=\mathbb{C} \oplus \mathfrak{g}, \quad \mathcal{V}^{\text {poiss }}\left(g l_{N}\right)=\mathbb{C} \oplus g l_{N}
$$

and vertex algebroids resp.

$$
\mathcal{V}(\mathcal{V} \text { ir })_{c}=\mathbb{C}, \quad \mathcal{V}(\mathfrak{g})_{k}=\mathbb{C} \oplus \mathfrak{g}, \quad \mathcal{V}\left(g l_{N}\right)_{k_{1}, k_{2}}=\mathbb{C} \oplus g l_{N}
$$

Here is an example of geometric nature.

Example 3.4. Let $A$ be a commutative associative algebra with unit 1, $\Omega(A)$ the module of Kähler differentials, $\operatorname{Der}(A)$ the algebra of derivations. Note that the homological degree grading of $A$ determines that of $\Omega(A)$ and $\operatorname{Der}(A)$. Set $V_{0}=A, V_{1}=\operatorname{Der}(A) \oplus \Omega(A)$, and let $T \stackrel{\text { def }}{=} d: V_{0}=A \rightarrow \Omega_{A} \hookrightarrow V_{1}$ be the canonical (de Rham) derivation. Then the space $V_{0} \oplus V_{1}$ carries a unique Courant algebroid structure determined by

$$
\begin{aligned}
& a_{(-1)} b=a b, \quad a_{(-1)}(\tau+\omega)=a \tau+a \omega, \quad \tau_{(0)} a=\tau(a), \\
& \tau_{(0)} \omega=\operatorname{Lie}_{\tau} \omega, \quad \tau_{(1)} \omega=\iota_{\tau} \omega, \\
& \tau_{(0)} \xi=[\tau, \xi], \tau_{(1)} \xi=0
\end{aligned}
$$

for all $a, b \in A, \omega \in \Omega(A), \tau, \xi \in \operatorname{Der}(A)$. Denote thus defined Courant algebroid by $\mathcal{V}^{\text {poiss }}(A)$.

Since all the operations recorded in (3.10) are of geometric nature, there arises, for each scheme $X$, a sheaf of vertex algebroids

$$
\mathcal{V}_{X}^{\text {poiss }} \stackrel{\text { def }}{=} \mathcal{T}_{X} \oplus \Omega_{X}
$$

Note that in keeping with our convention we assume that $X$ is graded, that is to say, $\mathcal{O}_{X}$ is a sheaf of graded algebras; consequently, $\mathcal{T}_{X}$ and $\Omega_{X}$ are sheaves of graded $\mathcal{O}_{X}$-modules.

If $\mathcal{V}=\mathcal{V}_{0} \oplus \mathcal{V}_{1}$ is a vertex algebroid, then part of its structure coincides with that of $\mathcal{V}^{\text {poiss }}(A)$. For example, the triple $\left(\mathcal{V}_{0},(-1), \mathbf{1}\right)$ is a commutative associative algebra with unit, $\left(\mathcal{V}_{0}\right)_{(-1)}\left(T\left(\mathcal{V}_{0}\right)\right)$ is a $\mathcal{V}_{0}$-module (although $\mathcal{V}_{1}$ is not), the map $T: \mathcal{V}_{0} \rightarrow\left(\mathcal{V}_{0}\right)_{(-1)}\left(T\left(\mathcal{V}_{0}\right)\right)$ is a derivation. There arises a filtration

$$
\mathcal{V}_{0} \oplus\left(\mathcal{V}_{0}\right)_{(-1)}\left(T\left(\mathcal{V}_{0}\right)\right) \subset \mathcal{V}
$$

and a moment's thought will show that, absolutely analogously to Section 2.7 , the corresponding Gr $\mathcal{V}$ carries a canonical Courant algebroid structure.

\section{Definition 3.5.}

(a) Given a Courant algebroid $\mathcal{V}^{\text {poiss }}$, call a vertex algebroid $\mathcal{V}$ a quantization of $\mathcal{V}^{\text {poiss }}$ if there is an isomorphism of Courant algebroids

$$
\operatorname{Gr} \mathcal{V} \stackrel{\sim}{\longrightarrow} \mathcal{V}^{\text {poiss }}
$$

(b) If $\mathcal{V}$ is a quantization of $\mathcal{V}^{\text {poiss }}(A)$, see Example 3.4, then we shall denote $\mathcal{V}$ by $\mathcal{V}(A)$.

Note that in the case of $\mathcal{V}^{\text {poiss }}(A)$, filtration (3.12) implies the following exact sequence of vector spaces

$$
0 \rightarrow \Omega(A) \rightarrow \mathcal{V}_{1} \stackrel{\pi}{\rightarrow} \operatorname{Der}(A) \rightarrow 0
$$


The problem of quantizing $\mathcal{V}^{\text {poiss }}(A)$ is not trivial and was studied in [15]. Call $A$ suitable for chiralization if $\operatorname{Der}(A)$ is a free $A$-module on generators $\tau_{1}, \ldots, \tau_{N}$ s.t. $\left[\tau_{i}, \tau_{j}\right]=0$. If $A$ is suitable for chiralization and a basis $\tau_{1}, \ldots, \tau_{N}$ is fixed, then $\mathcal{V}^{\text {poiss }}(A)$ can be quantized by letting $\mathcal{V}(A)=\mathcal{V}^{\text {poiss }}(A)$ as a vector space and requiring all of the relations (3.10) except the last two; the latter are to hold only for the basis vector fields:

$$
\left(\tau_{i}\right)_{(0)}\left(\tau_{j}\right)=0, \quad\left(\tau_{i}\right)_{(1)}\left(\tau_{j}\right)=0 .
$$

It is easy to show, using axioms (2.1)-(2.5), at least that these choices determine a vertex algebroid structure.

Let us point out the differences between the Courant and vertex algebroid structures thus obtained: in the Courant case operation (1) is an $A$-bilinear pairing and

$$
\left(f_{(-1)} \tau_{i}\right)_{(1)}\left(g_{(-1)} \tau_{j}\right)=0
$$

while in the vertex case

$$
\left(f_{(-1)} \tau_{i}\right)_{(1)}\left(g_{(-1)} \tau_{j}\right)=-f_{(-1)}\left(\tau_{j}\left(\tau_{i}(g)\right)-g_{(-1)}\left(\tau_{i}\left(\tau_{j}(f)\right)-\tau_{i}(g)_{(-1)} \tau_{j}(f) ;\right.\right.
$$

in the Courant case multiplication $(-1)$ is associative, e.g.,

$$
(f g)_{(-1)} \tau_{i}=f_{(-1)}\left(g_{(-1)} \tau_{i}\right)
$$

in the vertex case it is not as

$$
(f g)_{(-1)} \tau_{i}=f_{(-1)}\left(g_{(-1)} \tau_{i}\right)-\tau_{i}(g) d f-\tau_{i}(f) d g
$$

here $f, g \in A$ and axioms (2.3)-(2.5) along with simplifications due to grading have been used.

Furthermore, if $A$ is suitable for chiralization, then

\{isomorphism classes of quantizations of $\left.\mathcal{V}^{\text {poiss }}(A)\right\}$

$$
\text { is an }\left(\Omega^{3, \mathrm{cl}}(A) / d \Omega^{2}(A)\right)^{0}-\text { torsor, }
$$

and

$$
\operatorname{Aut}(\mathcal{V}(A)) \stackrel{\sim}{\longrightarrow}\left(\Omega^{2, \mathrm{cl}}(A)\right)^{0}
$$

where the automorphism of $\mathcal{V}(A)$ attached to $\omega \in \Omega^{2, \mathrm{cl}}(A)^{0}$ is defined by the assignment

$$
\mathcal{V}(A)_{1} \rightarrow \mathcal{V}(A)_{1}, \quad \tau \mapsto \tau+\omega(\pi(\tau)),
$$

$\pi$ being defined in (3.13). Note that we had to pay the price for relentlessly working in the graded setting by extracting the homological degree 0 subspace in (3.17), (3.18).

Since any smooth algebraic variety $X$ can be covered by the spectra of rings suitable for chiralization, (3.17), (3.18) create an avenue to define sheaves of vertex algebroids over $X$, to be denoted $\mathcal{V}_{X}$ or $\mathcal{V}_{A}$ if $X=\operatorname{Spec}(A)$.

Definition 3.6. Let $X$ be a graded scheme.

(a) Call a sheaf of vertex algebroids over $X$ a quantization of $\mathcal{V}_{X}^{\text {poiss }}$, see (3.11), if for each affine $U \subset X$ its space space of sections over $U$ is a quantization of $\Gamma\left(U, \mathcal{V}_{X}^{\text {poiss }}\right)$.

(b) Denote by $\mathcal{V e r t}_{X}\left(\right.$ or $\mathcal{V e r t}_{A}$ if $X=\operatorname{Spec}(A)$ ) the category of quantizations of the sheaf $\mathcal{V}_{X}^{\text {poiss }}$ 
The characteristic property of $\mathcal{V}_{X} \in \mathcal{V} \mathrm{ert}_{X}$ is the existence of the sequence of sheaves

$$
0 \rightarrow \Omega_{X} \rightarrow \mathcal{V}_{X} \stackrel{\pi}{\rightarrow} \mathcal{T}_{X} \rightarrow 0
$$

this is a sheaf analogue of (3.13).

Here are the (obvious graded versions of the) main results of [15]:

- there is a gerbe of vertex algebroids over a manifold $X$ such that the space of sections over each $U=\operatorname{Spec}(A) \subset X, A$ being suitable for chiralization, is the category of quantizations of $\mathcal{V}^{\text {poiss }}(A)$;

- in the case where $\mathcal{O}_{X}=\mathcal{O}_{X}^{0}$ this gerbe possesses a global section, i.e., a sheaf $\mathcal{V}_{X} \in \mathcal{V}_{\text {ert }}$, if and only if the 2nd component of the Chern character

$$
\operatorname{ch}_{2}\left(\mathcal{T}_{X}\right) \in H^{2}\left(X, \Omega_{X}^{2} \rightarrow \Omega_{X}^{3, \mathrm{cl}}\right)
$$

vanishes; if this class vanishes, then

$$
\left\{\text { Isomorphism classes of sheaves } \mathcal{V}_{X}\right\} \text { is an } H^{1}\left(X, \Omega_{X}^{2} \rightarrow \Omega_{X}^{3, \mathrm{cl}}\right)-\text { torsor; }
$$

- the forgetful functor (3.6) has a left adjoint functor

$$
\mathcal{U}_{\text {alg }}: \quad\{\text { Vertex algebroids }\} \rightarrow\{\text { Vertex algebras }\}
$$

if the obstruction (3.21) vanishes, we call

$$
D_{X}^{\text {ch }} \stackrel{\text { def }}{=} \mathcal{U}_{\text {alg }} \mathcal{V}_{X}
$$

a sheaf of chiral differential operators, CDO for short.

Note that proving (3.22) amounts to covering $X$ by open sets that are suitable for chiralization and re-gluing a given sheaf by composing the old gluing functions with automorphisms (3.18), (3.19).

\subsection{Further examples and constructions.}

3.7.1. Localization. For any quantization $\mathcal{V}(A)$ and an ideal $\mathfrak{a} \subset A$ a natural quantization $\mathcal{V}\left(A_{\mathfrak{a}}\right)$ is defined [15], the reason being that all the ${ }_{(n)}$-products on $\mathcal{V}(A)$ are in fact certain differential operators. For example, at the quasiclassical level, all the operations recorded in (3.10) are differential operators of order $\leq 1$. Furthermore, (3.15), (3.16) provide examples of genuine quantum operations being order $\leq 2$ differential operators. Therefore, given an $A$ and $\mathcal{V}(A)$, there arises a sheaf of vertex algebroids

$$
\mathcal{V}_{A} \in \mathcal{V} \operatorname{ert}_{A} \quad \text { s.t. } \quad \Gamma\left(\operatorname{Spec}(A), \mathcal{V}_{A}\right)=\mathcal{V}(A)
$$

This construction underlies the above discussion of gerbes of vertex algebroids.

A little more generally, if $X$ and $Y$ are manifolds and $p: X \rightarrow Y$ is a covering, then there is a functor

$$
p^{*}: \operatorname{Vert}_{Y} \rightarrow \operatorname{Vert}_{X}
$$

The reason for this to be true is that the story of quantizing $\mathcal{V}^{\text {poiss }}(A), \operatorname{Spec}(A) \subset Y$, starts with a choice of an Abelian basis $\left\{\tau_{i}\right\} \subset \operatorname{Der}(A)$, and any such choice is canonically lifted to an Abelian basis of $\Gamma\left(V, \mathcal{T}_{X}\right)$ for any affine $V \subset p^{-1}(\operatorname{Spec}(A))$. 
In particular, if $X$ carries a free action of a finite group $G$, then there arises an equivalence of categories

$$
\mathcal{V e r t}_{X / G} \rightarrow \mathcal{V} \operatorname{rrt}_{X}^{G}
$$

where $\mathcal{V e r t}_{X}^{G}$ is a full subcategory of $G$-equivariant vertex algebroids. The inverse functor is, essentially, that of $G$-invariants

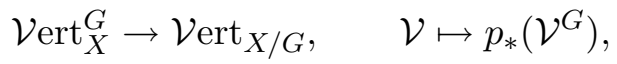

where $p_{*}$ is the push-forward in the category of sheaves of vector spaces.

Here is a version of localization called a push-out in $[15,16]$. If a Lie group acts on $A$ by derivations, then

$$
A \otimes \mathcal{V}(\mathfrak{g})_{k} \stackrel{\text { def }}{=} A \oplus A \otimes \mathfrak{g} \oplus \Omega(A)
$$

is a vertex algebroid.

3.7.2. Affine space. According to (3.21), (3.22), there is a unique sheaf of vertex algebroids over $\mathbb{C}^{N}, \mathcal{V}_{\mathbb{C}^{N}}$. Its space of global sections is $\mathcal{V}\left(\mathbb{C}\left[x_{1}, \ldots, x_{N}\right]\right)$, where we take $\left\{\partial_{j}=\partial / \partial x_{j}\right\}$ for an Abelian basis of $\operatorname{Der}\left(\mathbb{C}\left[x_{1}, \ldots, x_{N}\right]\right)$, and if $U=\{f \neq 0\}$, then $\Gamma\left(U, \mathcal{V}_{\mathbb{C}_{N}}\right)$ is defined via the localization of Section 3.7.1.

The corresponding CDO, see $(3.24)$, is $D_{\mathbb{C}^{N}}^{\text {ch }}=\mathcal{U}_{\text {alg }} \mathcal{V}_{\mathbb{C}^{N}}$. Its space of global sections, denoted by either $D^{\mathrm{ch}}\left(\mathbb{C}^{N}\right)$ or $D^{\mathrm{ch}}\left(\mathbb{C}\left[x_{1}, \ldots, x_{N}\right]\right)$, is likewise obtained via $\mathcal{U}_{\text {alg }}: D^{\mathrm{ch}}\left(\mathbb{C}^{N}\right)=$ $\mathcal{U}_{\text {alg }} \mathcal{V}\left(\mathbb{C}\left[x_{1}, \ldots, x_{N}\right]\right)$. More explicitly, $D^{\text {ch }}\left(\mathbb{C}^{N}\right)$ can be defined to be the vertex algebra generated freely by the vector space $\mathbb{C}^{N} \oplus\left(\mathbb{C}^{N}\right)^{*}$ and relations

$$
\left(\partial_{i}\right)_{(0)} x_{j}=-\left(x_{j}\right)_{(0)} \partial_{i}=\delta_{i j} \mathbf{1}, \quad a_{(n)} b=0 \quad \text { for all } \quad a, b \in \mathbb{C}^{N} \oplus\left(\mathbb{C}^{N}\right)^{*}, \quad n>0,
$$

where $\left\{\partial_{i}\right\},\left\{x_{j}\right\}$ are dual bases of $\mathbb{C}^{N}$ and $\left(\mathbb{C}^{N}\right)^{*}$ resp.

3.7.3. Punctured plane. Let $X=\mathbb{C}^{2} \backslash(0,0)$ and choose the trivial grading where $\mathcal{O}_{X}=\mathcal{O}_{X}^{0}$. The obstruction (3.21) vanishes, because one sheaf, say, the restriction of $\mathcal{V}_{\mathbb{C}^{2}}$ to $\mathbb{C}^{2} \backslash(0,0)$, exists. Isomorphism classes of sheaves of vertex algebroids over $X$ are easy to classify. Indeed, consider the affine covering

$$
X=U_{1} \cup U_{2}, \quad U_{j}=\left\{\left(y_{1}, y_{2}\right) \text { s.t. } y_{j} \neq 0\right\}
$$

and the Cech 1-cocycle

$$
\omega_{a b}: \quad U_{1} \cap U_{2} \mapsto \frac{d y_{1} \wedge d y_{2}}{y_{1}^{a} y_{2}^{b}}, \quad a, b \geq 1 .
$$

It is known (and easy to check) that

$$
H^{1}\left(X, \Omega_{X}^{2} \rightarrow \Omega_{X}^{3, \mathrm{cl}}\right)=\oplus_{a, b} \mathbb{C} \omega_{a b}
$$

Hence the isomorphism classes of sheaves of vertex algebroids over $X$ are in 1-1 correspondence with linear combinations of $\omega_{a b}$. Here is an explicit construction of the sheaf attached to $k \omega_{a b}$ : let $\mathcal{V}_{X}$ be the restriction of the standard $\mathcal{V}_{\mathbb{C}^{2}}$ to $X, \mathcal{V}_{U_{j}}$ its pull-back to $U_{j}, j=1,2$; now glue $\mathcal{V}_{U_{1}}$ and $\mathcal{V}_{U_{2}}$ over the intersection $U_{1} \cap U_{2}$, cf. (3.18), (3.19), as follows:

$$
\partial_{1} \rightarrow \partial_{1}+\frac{k T\left(y_{2}\right)}{y_{1}^{a} y_{2}^{b}}, \quad \partial_{2} \rightarrow \partial_{2}-\frac{k T\left(y_{1}\right)}{y_{1}^{a} y_{2}^{b}} .
$$


It is immediate to generalize this to the case of an arbitrary $\omega$ in the linear span of $\left\{\omega_{a b}\right\}$. Denote the sheaf sheaf thus defined by $\mathcal{V}_{X, \omega}$.

This simple example will be essential for our purposes.

3.7.4. Symmetries. Given a Lie algebra morphism

$$
\rho: \quad \mathfrak{g} \rightarrow \operatorname{Der}(A),
$$

the composition

$$
\mathfrak{g} \rightarrow \operatorname{Der}(A) \stackrel{(\mathrm{id}, 0)}{\hookrightarrow} \mathcal{V}^{\text {poiss }}(A)=\operatorname{Der}(A) \oplus \Omega(A)
$$

defines a Courant algebroid morphism

$$
\rho^{\text {poiss }}: \quad \mathcal{V}^{\text {poiss }}(\mathfrak{g}) \rightarrow \mathcal{V}^{\text {poiss }}(A)
$$

Furthermore, any Poisson algebroid morphism $\rho^{\text {poiss }}$, upon quotienting out by $\Omega(A)$, defines a Lie algebra morphism $\rho$.

A quantization of a Courant algebroid morphism $\rho^{\text {poiss }}: \mathcal{V}^{\text {poiss }}(\mathfrak{g}) \rightarrow \Gamma\left(X, \mathcal{V}_{X}^{\text {poiss }}\right)$ is defined to be a vertex algebroid morphism $\hat{\rho}: \mathcal{V}(\mathfrak{g})_{k} \rightarrow \mathcal{V}(A)$ such that the diagram

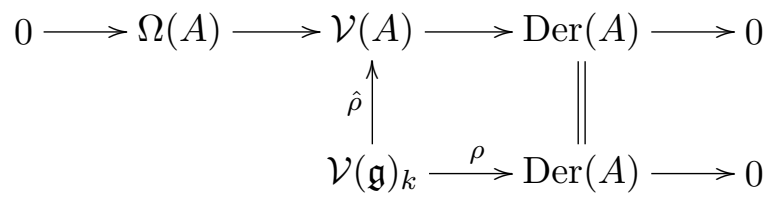

commutes. Here the arrow $\mathcal{V}(\mathfrak{g})_{k} \stackrel{\rho}{\rightarrow} \operatorname{Der}(A)$ means the composition $\mathcal{V}(\mathfrak{g})_{k}=\mathbb{C} \oplus \mathfrak{g} \stackrel{0 \oplus \rho}{\longrightarrow} \operatorname{Der}(A)$, see (3.9).

Similarly, if $\mathfrak{g}$ operates on $X$, that is, there is a Lie algebra morphism

$$
\rho: \quad \mathfrak{g} \rightarrow \Gamma\left(X, \mathcal{T}_{X}\right)
$$

then a quantization of the corresponding Courant algebroid morphism $\rho^{\text {poiss }}: \mathcal{V}^{\text {poiss }}(\mathfrak{g}) \rightarrow$ $\Gamma\left(X, \mathcal{V}_{X}^{\text {poiss }}\right)$ is defined to be a vertex algebroid morphism $\hat{\rho}: \mathcal{V}(\mathfrak{g})_{k} \rightarrow \Gamma\left(X, \mathcal{V}_{X}\right)$ such that the diagram

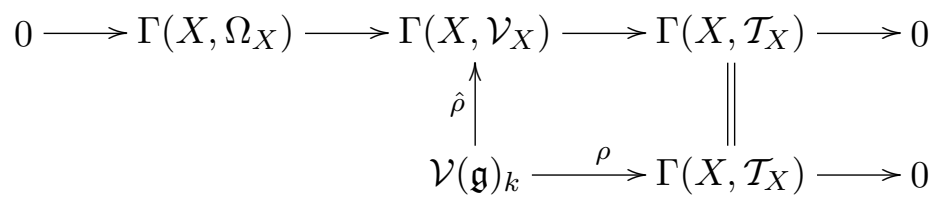

commutes.

To see an example of importance for what follows, let us consider the tautological action of $g l_{2}$ on $\mathbb{C}^{2}$. If we let $X=\mathbb{C}^{2} \backslash(0,0)$, then there arises

$$
\rho^{\text {poiss }}: \quad \mathcal{V}^{\text {poiss }}\left(g l_{2}\right) \rightarrow \Gamma\left(\mathbb{C}^{2} \backslash(0,0), \mathcal{V}_{\mathbb{C}^{2} \backslash(0,0)}^{\text {poiss }}\right)
$$

and we ask if this map can be quantized to a map $\mathcal{V}\left(g l_{2}\right)_{k_{1}, k_{2}} \rightarrow \Gamma\left(\mathbb{C}^{2} \backslash(0,0), \mathcal{V}_{\mathbb{C}^{2} \backslash(0,0), \omega}\right)$, where the vertex algebroid $\mathcal{V}_{\mathbb{C}^{2} \backslash(0,0), \omega}$ was defined in Section 3.7.3. 
Lemma 3.7.5. Quantization of (3.32),

$$
\hat{\rho}: \quad \mathcal{V}\left(g l_{2}\right)_{k_{1}, k_{2}} \rightarrow \Gamma\left(\mathbb{C}^{2} \backslash(0,0), \mathcal{V}_{\mathbb{C}^{2} \backslash(0,0), \omega}\right)
$$

exists if and only if $\omega=k d y_{1} \wedge d y_{2} / y_{1} y_{2}, k_{1}=-k-1, k_{2}=k-1$ for some $k \in \mathbb{C}$.

Proof. We shall use the notation of Section 3.7.3. In terms of the coordinates $y_{1}, y_{2}$ the morphism $\rho$ is this

$$
\rho\left(E_{i j}\right) \mapsto y_{i} \partial_{j}
$$

If we consider $y_{i} \partial_{j}$ as an element of $\Gamma\left(U_{1}, \mathcal{V}_{X, \omega}\right)$, then over $U_{2}$ it becomes, according to (3.29), $\left(y_{i} \partial_{j} \pm k T\left(y_{j \pm 1}\right) y_{i}\right) / y_{1}^{a} y_{2}^{b}$ and hence may develop a pole. To compensate for it, we can choose a different lift of $E_{i j}$ to $\Gamma\left(U_{1}, \mathcal{V}_{X, \omega}\right)$ by replacing $y_{i} \partial_{j}$ with $y_{i} \partial_{j}+\alpha$, where $\alpha \in \Gamma\left(U_{1}, \Omega_{X}\right)$. Over $U_{2}$ this element becomes $\left(y_{i} \partial_{j} \pm T\left(y_{j \pm 1}\right) y_{i}\right) / y_{1}^{a} y_{2}^{b}+\alpha$. Since $\alpha$ may have at most a pole along $\left\{y_{1}=0\right\}$, for this element to extend to a section over $U_{2}$, one of the following two things must happen: either $T\left(y_{j \pm 1}\right) y_{i} / y_{1}^{a} y_{2}^{b}$ has no pole along $\left\{y_{1}=0\right\}$, in which case no $\alpha$ is needed, or $T\left(y_{j \pm 1}\right) y_{i} / y_{1}^{a} y_{2}^{b}$ has no pole along $\left\{y_{2}=0\right\}$, in which case a desired $\alpha$ can be found. For a favorable event to occur for $i=1$ and $i=2$, both $a$ and $b$ must be at most 1 . But by definition, see Section 3.7.3, $a$ and $b$ are at least 1; therefore a linear map $g l_{2} \rightarrow \Gamma\left(X, \mathcal{V}_{X, \omega}\right)$ may exist only if $\omega=k d y_{1} \wedge d y_{2} / y_{1} y_{2}$. On the other hand, if $\omega=k d y_{1} \wedge d y_{2} / y_{1} y_{2}$, then the map $\hat{\rho}$ defined so that

$$
\begin{aligned}
& \hat{\rho}\left(E_{12}\right)=y_{1} \partial_{y_{2}}, \\
& \hat{\rho}\left(E_{21}\right)=y_{2} \partial_{y_{1}}-\frac{k y_{2}^{\prime}}{y_{1}}, \\
& \hat{\rho}\left(E_{11}\right)=y_{1} \partial_{y_{1}}, \\
& \hat{\rho}\left(E_{22}\right)=y_{2} \partial_{y_{2}}+\frac{k y_{1}^{\prime}}{y_{1}} .
\end{aligned}
$$

delivers the desired vertex algebroid morphism

$$
\hat{\rho}: \quad \mathcal{V}\left(g l_{2}\right)_{-k-1, k-1} \rightarrow \Gamma\left(\mathbb{C}^{2} \backslash(0,0), \mathcal{V}_{\mathbb{C}^{2} \backslash(0,0), \omega}\right), \quad \omega=k d y_{1} \wedge d y_{2} / y_{1} y_{2}
$$

It is easy to see the vertex algebroid morphism condition determines the map uniquely.

Note that the top row of (3.31), unlike that of (3.30), does not have to be exact in general. In the case at hand, however, it is precisely when $\omega=k \omega_{11}$ :

Corollary 3.7.6. The sequence

$$
0 \rightarrow \Gamma\left(\mathbb{C}^{2} \backslash(0,0), \Omega_{\mathbb{C}^{2} \backslash(0,0)}\right) \rightarrow \Gamma\left(\mathbb{C}^{2} \backslash(0,0), \mathcal{V}_{\mathbb{C}^{2} \backslash(0,0), \omega}\right)_{1} \rightarrow \Gamma\left(\mathbb{C}^{2} \backslash(0,0), \mathcal{T}_{\mathbb{C}^{2} \backslash(0,0)}\right) \rightarrow 0
$$

is exact if and only if $\omega=k \omega_{11}$ for some $k \in \mathbb{C}$.

Proof. Notice that $\mathcal{T}_{\mathbb{C}^{2} \backslash(0,0)}$ is generated by $\rho\left(g l_{2}\right)$ over functions. The "if" part is then seen to be an immediate consequence of Lemma 3.7.5. The "only if" part was actually proved at the beginning of the proof of the lemma cited. 
3.7.7. Conformal structure. If $x_{1}, \ldots, x_{N}$ are coordinates on $\mathbb{C}^{N}, \partial_{j}=\partial / \partial_{j}$, then there is a vertex (Poisson) algebra morphism

$$
\begin{aligned}
& \mathcal{U}^{\text {poiss }} \mathcal{L}(\mathcal{V} \text { ir }) \rightarrow \Gamma\left(\mathbb{C}^{N}, \mathcal{D}_{\mathbb{C}^{N}}^{\text {poiss }}\right), \quad \mathcal{U} \mathcal{L}(\mathcal{V} \text { ir })_{2 N} \rightarrow \Gamma\left(\mathbb{C}^{N}, \mathcal{D}_{\mathbb{C}^{N}}\right), \\
& L \mapsto \sum_{j=1}^{N} T\left(x_{j}\right)_{(-1)} \partial_{j},
\end{aligned}
$$

where the latter is a quantization of the former. A little more generally, if $A$ is an algebra suitable for chiralization with $\tau_{1}, \ldots, \tau_{N}$ an Abelian basis of $\operatorname{Der}(A)$, then one can find a coordinate system, i.e., $\left\{x_{1}, \ldots, x_{N}\right\} \subset A$ s.t. $\tau_{i}\left(x_{j}\right)=\delta_{i j}$, and thus obtain

$$
\mathcal{U}^{\text {poiss }} \mathcal{L}(\mathcal{V} \text { ir }) \rightarrow \mathcal{U}^{\text {poiss }} \mathcal{V}^{\text {poiss }}(A), \quad \mathcal{U} \mathcal{L}(\mathcal{V} \text { ir })_{2 N} \rightarrow \mathcal{U} \mathcal{V}(A), \quad L \mapsto \sum_{j=1}^{N} T\left(x_{j}\right)_{(-1)} \tau_{j}
$$

In this case, $N$ is the Krull dimension of $A$.

Another example is provided by the twisted sheaves $\mathcal{V}_{\mathbb{C}^{2} \backslash 0, \omega}$ of Section 3.7.3. Somewhat unexpectedly, the same definition (3.39), which in the present case becomes $L \mapsto T\left(y_{1}\right) \partial_{1}+T\left(y_{2}\right) \partial_{2}$, applied locally over both both charts $U_{1}$ and $U_{2}$ survives the twisted gluing transformation (3.29) for any $\omega$ and defines a global morphism

$$
\mathcal{U L}(\mathcal{V} \text { ir })_{2} \rightarrow \Gamma\left(\mathbb{C}^{2} \backslash 0, \mathcal{V}_{\mathbb{C}^{2} \backslash 0, \omega}\right)
$$

\section{A graded Courant algebroid attached to a commutative associative algebra}

4.1. Modules of differentials. Even though the assumption that all the vector spaces in question are $\mathbb{Z}$-graded has been kept since the very beginning of Section 2, it has been barely used. From now on it will be essential and referred to explicitly as in the following definition.

Definition 4.1.1. A differential graded algebra (DGA) $R$ is a pair $\left(R_{\#}, D\right)$, where $R_{\#}=$ $\oplus_{n=0}^{\infty} R^{n}$ is a graded supercommutative associative algebra with $R^{\text {even }}=\oplus_{n=0}^{\infty} V^{2 n}, R^{\text {odd }}=$ $\oplus_{n=0}^{\infty} R^{2 n+1}$, and $D$ is a square 0 degree $(-1)$ (hence odd) derivation. Call a DGA $\left(R_{\#}, D\right)$ quasi-free if there is a graded vector superspace $V=\oplus_{n=0}^{\infty} V^{n}$ with $V^{\text {even }}=\oplus_{n=0}^{\infty} V^{2 n}$, $V^{\text {odd }}=$ $\oplus_{n=0}^{\infty} V^{2 n+1}$ such that $R_{\#}$ is the symmetric algebra $S^{\bullet} V$.

If $R$ is a DGA, then $H_{D}^{\bullet}(R) \stackrel{\text { def }}{=} \operatorname{Ker}(D) / \operatorname{Im}(D)$ is a a graded supercommutative associative algebra.

For any commutative associative algebra $A$ there is a quasi-free DGA $R$ and a quasi-isomorphism

$$
R \rightarrow A,
$$

that is to say, a DGA morphism ( $A$ being placed in homological degree 0 and equipped with a zero differential) that delivers a graded algebra isomorphism $H_{D}^{\bullet}(R) \stackrel{\sim}{\longrightarrow} A$.

If $A$ is finitely generated, then a DGA resolution $R$ can be chosen so that each $V^{j}$ from Definition 4.1.1 is finite dimensional. These two finiteness assumptions will be made throughout.

A DGA resolution of $A$ is not unique, but for any two such resolutions

$$
R_{1} \rightarrow A \leftarrow R_{2}
$$


there is a homotopy equivalence [2]

$$
f: \quad R_{1} \rightarrow R_{2} .
$$

If $R$ is a quasi-free DGA, denote by $\Omega(R)$ the module of Kähler differentials of $R$. It is canonically a differential-graded (DG) free $R$-module with derivation $d: R \rightarrow \Omega(R)$ and differential Lie $_{D}$, which we choose to denote by $D$, too. The correspondence $R \mapsto \Omega(R)$ is functorial in that naturally associated to an algebra morphism $f: R_{1} \rightarrow R_{2}$ there is a map of DG $R_{1}$-modules:

$$
\Omega(f): \quad \Omega\left(R_{1}\right) \rightarrow \Omega\left(R_{2}\right) .
$$

Furthermore, we have

$$
\Omega(R)=\bigoplus_{n=0}^{+\infty} \Omega(R)^{n}, \quad d: R^{n} \rightarrow \Omega(R)^{n}, \quad D: \Omega(R)^{n} \rightarrow \Omega(R)^{n-1}, \quad[d, D]=0 .
$$

It follows that the homology $H_{D}(\Omega(R))$ is naturally a graded $H_{D}^{\bullet}(R)$-module.

For any 2 quasi-free DGA resolutions $R_{1} \rightarrow A \leftarrow R_{2}$, we can find a homotopy equivalence $f: R_{1} \rightarrow R_{2}$, see (4.2), hence a quasi-isomorphism

$$
\Omega(f): \Omega\left(R_{1}\right) \rightarrow \Omega\left(R_{2}\right)
$$

and an isomorphism

$$
H(\Omega(f)): \quad H_{D_{1}}^{\bullet}\left(\Omega\left(R_{1}\right)\right) \rightarrow H_{D_{2}}^{\bullet}\left(\Omega\left(R_{2}\right)\right) .
$$

\section{Definition 4.1.2.}

$$
\Omega(A)^{\bullet}=H_{D}^{\bullet}(\Omega(R)),
$$

where $R$ is a quasi-free DGA resolution of $A$.

The assignment $A \mapsto \Omega(A)^{\bullet}$ defines a functor from the category of algebras to the category of graded vector spaces.

Note that

$$
\Omega(A)^{\bullet}=\bigoplus_{n=0}^{+\infty} \Omega(A)^{n}
$$

is a graded $A$-module, and $\Omega(A)^{0}$ is the module of Kähler differentials of $A, \Omega(A)$.

4.2. Modules of derivations. If $R$ is a quasi-free DGA, we denote by $\operatorname{Der}(R)$ the Lie algebra of derivations of $R$. Like $\Omega(R)$, it is a DG $R$-module, but unlike $\Omega(R)$ it is graded in both directions:

$$
\operatorname{Der}(R)=\bigoplus_{n \in \mathbb{Z}} \operatorname{Der}(R)^{n}
$$

and, which is more serious, not free; in fact, each component $\operatorname{Der}(R)^{n}$ is a direct product

$$
\operatorname{Der}(R)^{n}=\prod_{j=0}^{+\infty}\left(V^{j}\right)^{*} \otimes R^{n+j}
$$

where $V^{j}$ is one of the ingredients of Definition 4.1.1 assumed to be finite dimensional. 
The derivation $[D, \cdot]: \operatorname{Der}(R) \rightarrow \operatorname{Der}(R)$ is a differential because $D \in \operatorname{Der}(R)^{-1}$ is odd. Hence a Lie algebra $H_{[D, \cdot]}^{\bullet}(\operatorname{Der}(R))$ arises.

The assignment $R \mapsto \operatorname{Der}(R)$ is not quite functorial, because even if $f: R_{1} \rightarrow R_{2}$ is a quasiisomorphism, a Lie algebra morphism $\operatorname{Der}(f): \operatorname{Der}\left(R_{1}\right) \rightarrow \operatorname{Der}\left(R_{2}\right)$ does not quite exist. It does exist though at the level of the corresponding homotopy categories. This remark and what follows belongs to Hinich [18, Section 8].

Decompose $f: R_{1} \rightarrow R_{2}$ as follows

$$
f: \quad R_{1} \stackrel{i}{\hookrightarrow} S \stackrel{p}{\rightarrow} R_{2},
$$

where $i$ is a standard acyclic cofibration, and $p$ is an acyclic fibration. (Recall that $i$ being a standard cofibration means $S$ being obtained by adjoining variables to $R_{1}$, and being a fibration means being an epimorphism.)

In the case of $i$, there arises a diagram of quasiisomorphisms

$$
\operatorname{Der}\left(R_{1}\right) \stackrel{\pi_{i}}{\leftarrow} \operatorname{Der}(i) \stackrel{\mathrm{in}_{i}}{\longrightarrow} \operatorname{Der}(S),
$$

where $\operatorname{Der}(i)=\left\{\tau \in \operatorname{Der}(S)\right.$ s.t. $\left.\tau\left(R_{1}\right) \subset R_{1}\right\}, \pi_{i}$ is the obvious projection, and $\operatorname{in}_{i}$ is the obvious embedding.

Analogously, in the case of $p$, there is a diagram of quasiisomorphisms

$$
\operatorname{Der}(S) \stackrel{\operatorname{in}_{p}}{\longleftarrow} \operatorname{Der}(p) \stackrel{\pi_{p}}{\rightarrow} \operatorname{Der}(S),
$$

where $\operatorname{Der}(p)=\left\{\tau \in \operatorname{Der}\left(R_{1}\right)\right.$ s.t. $\left.\tau(\operatorname{Ker}(p)) \subset \operatorname{Ker}(p)\right\}$, $\mathrm{in}_{p}$ is the obvious embedding, and $\pi_{p}$ is the obvious projection.

Hinich defines

$$
\operatorname{Der}(i)=\operatorname{in}_{i} \circ \pi_{i}^{-1}, \quad \operatorname{Der}(p)=\pi_{p} \circ \operatorname{in}_{p}^{-1}, \quad \operatorname{Der}(f)=\operatorname{Der}(p) \circ \operatorname{Der}(i) .
$$

This map makes sense in the homotopy category and delivers a homotopy category quasiisomorphism

$$
\operatorname{Der}(f): \operatorname{Der}\left(R_{1}\right) \rightarrow \operatorname{Der}\left(R_{2}\right) .
$$

Hence an isomorphism

$$
H(\operatorname{Der}(f)): \quad H_{\left[D_{1}, \cdot\right]}^{\bullet}\left(R_{1}\right) \rightarrow H_{\left[D_{2}, \cdot\right]}^{\bullet}\left(R_{2}\right) .
$$

Theorem 4.2.1 ([18]). If $f_{j}: R_{1} \rightarrow R_{2}, j=1,2$, are homotopic to each other, then $\operatorname{Der}\left(f_{j}\right)$, $j=1,2$, are also.

\section{Corollary 4.2.2 ([18]).}

(i) The assignment $R \mapsto \operatorname{Der}(R)$ defines a functor from the homotopy category of DG commutative associative algebras with quasi-isomorphisms to the homotopy category of DG Lie algebras.

(ii) The assignment $R \mapsto H_{[D, \cdot}^{\bullet}(\operatorname{Der}(R))$ defines a functor from the homotopy category of $D G$ commutative associative algebras with quasi-isomorphisms to the category of graded Lie algebras.

\section{Definition 4.2.3.}

$$
\operatorname{Der}(A)^{\bullet}=H_{[D, \cdot]}^{\bullet}(\operatorname{Der}(R)),
$$

where $R$ is a quasi-free DGA resolution of $A$. 
4.3. Synthesis: Courant algebroids. The notion of a Courant algebroid allows us to bring Sections 4.1 and 4.2 under the same roof. Let $\mathcal{V}^{\text {poiss }}$ be a Courant algebroid. It follows from the Jacobi identity (2.4) that, for any $\xi \in \mathcal{V}^{\text {poiss }}(R), \xi_{(0)} \in \operatorname{End}\left(\mathcal{V}^{\text {poiss }}(R)\right)$ is a derivation of all products. Identity (2.2) implies that $\xi_{(0)}$ commutes with $T$. If, in addition, $\xi$ is odd and $\xi_{(0)} \xi=0$, then $\left(\xi_{(0)}\right)^{2}=0$ as another application of $(2.4)$ shows. Therefore, a pair $\left(\mathcal{V}^{\text {poiss }}, \xi\right)$ is a differential Courant algebroid, and the homology Courant algebroid, $H_{\xi_{(0)}}^{\bullet}\left(\mathcal{V}^{\text {poiss }}\right)$, arises.

Let us now specialize this well-known construction to the Courant algebroid $\mathcal{V}^{\text {poiss }}(R)=$ $\operatorname{Der}(R) \oplus \Omega(R)$, see Example 3.4, in the case of a quasi-free DGA $R=\left(R_{\#}, D\right)$. By definition $D$ is odd and, according to $(3.10), D_{(0)} D=[D, D]=0$. Hence the pair $\left(\mathcal{V}^{\text {poiss }}(R), D_{(0)}\right)$ is a DG Courant algebroid and the graded Courant algebroid $H_{D_{(0)}}^{\bullet}\left(\mathcal{V}^{\text {poiss }}(R)\right)$ arises. Again by virtue of (3.10), the differential $D_{(0)}$ preserves $\operatorname{Der}(R) \subset \mathcal{V}^{\text {poiss }}(R)$, where it coincides with $[D, \cdot]$, and $\Omega(R)$, where it coincides with the standard action of $D$ by the Lie derivative, see also (4.4). We obtain a canonical vector space isomorphism

$$
H_{D_{(0)}}^{\bullet}\left(\mathcal{V}^{\text {poiss }}(R)\right)=H_{[D, \cdot]}^{\bullet}(\operatorname{Der}(R)) \oplus H_{D}^{\bullet}(\Omega(R))
$$

If $f: R_{1} \rightarrow R_{2}$ is a homotopy equivalence, then

$$
H(\operatorname{Der}(f)) \oplus H(\Omega(f)): \quad H_{D_{(0)}}^{\bullet}\left(\mathcal{V}^{\text {poiss }}\left(R_{1}\right)\right) \rightarrow H_{D_{(0)}}^{\bullet}\left(\mathcal{V}^{\text {poiss }}\left(R_{2}\right)\right),
$$

is a vector space isomorphism by virtue of (4.6) and (4.16). In fact, (4.18) is a graded Courant algebroid isomorphism. This follows from the fact that the Courant algebroid structure on $\mathcal{V}^{\text {poiss }}(R)$ consists of classical differential geometry operations, such as the tautological action of $\operatorname{Der}(R)$ on $R$ and the action of $\operatorname{Der}(R)$ on $\Omega(R)$ by means of the Lie derivative. An inspection of maps (4.11)-(4.16) shows that Hinich's construction respects all these operations.

Corollary 4.3.1. The assignment $R \mapsto H_{D_{(0)}}^{\bullet}\left(\mathcal{V}^{\text {poiss }}(R)\right)$ defines a functor from the homotopy category of DG commutative associative algebras with quasi-isomorphisms to the category of graded Courant algebroids.

\section{Definition 4.3.2.}

$$
\mathcal{V}^{\text {poiss }}(A)^{\bullet}=H_{D_{(0)}}^{\bullet}\left(\mathcal{V}^{\text {poiss }}(R)\right)
$$

where $R$ is a quasi-free DGA resolution of $A$.

4.4. Conformal structure. The construction of Section 3.7.7 in the present setting means the following. If $R_{\#}=S^{\bullet} V$, pick a homogeneous basis $\left\{x_{i}\right\} \subset V$ and a dual 'basis' $\left\{\partial_{i}\right\} \subset V^{*}$, where $\partial_{i}\left(x_{j}\right)=\delta_{i j}$. As in Section 3.7.7, we obtain a morphism

$$
\mathcal{U}^{\text {poiss }} \mathcal{L}(\mathcal{V} \text { ir }) \rightarrow \mathcal{U V}^{\text {poiss }}(R), \quad L \mapsto \sum_{j}\left(T\left(x_{j}\right)\right)_{(-1)} \partial_{j}
$$

\section{Lemma 4.4.1.}

$$
\xi_{(0)} \sum_{j} T\left(x_{j}\right)_{(-1)} \partial_{j}=0 \quad \text { for any } \quad \xi \in \operatorname{Der}(R) .
$$


Corollary 4.4.2. Assignment (4.19) determines a Courant algebroid morphism

$$
\mathcal{U}^{\text {poiss }} \mathcal{L}(\mathcal{V} \text { ir }) \rightarrow \mathcal{U} \mathcal{V}^{\text {poiss }}(A)^{0}
$$

Proof of Lemma. Let $\xi=\sum_{i}\left(f_{i}\right)_{(-1)} \partial_{i}, f_{i} \in R_{\#}$. We have

$$
\begin{aligned}
\xi_{(0)} \sum_{j} T\left(x_{j}\right)_{(-1)} \partial_{j} & =\sum_{j}\left(\xi_{(0)} T\left(x_{j}\right)\right)_{(-1)} \partial_{j}+\sum_{j}(-1)^{\xi \cdot x_{j}} T\left(x_{j}\right)_{(-1)}\left(\xi_{(0)} \partial_{j}\right) \\
& =\sum_{j} T\left(\xi_{(0)} x_{j}\right)_{(-1)} \partial_{j}-\sum_{j}(-1)^{\xi \cdot x_{j}+\xi \cdot x_{j}} T\left(x_{j}\right)_{(-1)}\left(\left(\partial_{j}\right)_{(0)} \sum_{i}\left(f_{i}\right)_{(-1)} \partial_{i}\right) \\
& =\sum_{j} T\left(f_{j}\right)_{(-1)} \partial_{j}-\sum_{i} \sum_{j} T\left(x_{j}\right)_{(-1)}\left(\frac{\partial f_{i}}{\partial x_{j}}\right)_{(-1)} \partial_{i} \\
& =\sum_{j} T\left(f_{j}\right)_{(-1)} \partial_{j}-\sum_{i} T\left(f_{i}\right)_{(-1)} \partial_{i}=0 .
\end{aligned}
$$

4.5. Grading of $\operatorname{Der}(\boldsymbol{A})^{\bullet}$ and identification of $\operatorname{Der}(\boldsymbol{A})^{\mathbf{0}}$. Recall that $R$, hence $\Omega(R)$ and $\Omega(A)^{\bullet}$, are all graded by $\mathbb{Z}_{+}$. Contrary to this, although the Lie algebra $\operatorname{Der}(R)$ is graded in both directions, $\operatorname{Der}(A)$ is $\mathbb{Z}_{-}$-graded.

\section{Lemma 4.5.1.}

(a) $\operatorname{Der}(A)^{n}=0$ if $n \geq 0$;

(b) $\operatorname{Der}(A)^{0}$ is the Lie algebra of derivations of $A$.

Proof. Consider a quasi-free DGA resolution $R \rightarrow A$. The complex $(\operatorname{Der}(R),[D, \cdot])$ is filtered as follows, cf. (4.10),

$$
F^{p} \operatorname{Der}(R)^{n}=\prod_{j=p}^{+\infty}\left(V^{j}\right)^{*} \otimes R^{n+j}
$$

A spectral sequence $\left(E_{p q}^{r}, d_{r}\right) \Rightarrow \operatorname{Der}(A)^{p+q}$ arises so that

$$
\left(E_{p q}^{0}, d_{0}\right)=\left(\left(V^{-p}\right)^{*} \otimes R^{q}, 1 \otimes D\right) .
$$

Since $R=\left(R_{\#}, D\right)$ is quasi-isomorphic to $A$ placed in degree 0 , we have

$$
E_{p q}^{1}= \begin{cases}\left(V^{-p}\right)^{*} \otimes A & \text { if } q=0 \\ 0 & \text { otherwise. }\end{cases}
$$

It follows at once that the spectral sequence collapses and $\operatorname{Der}(A)^{-n}$ is the $n$-th cohomology of the complex

$$
0 \rightarrow\left(V^{0}\right)^{*} \otimes A \rightarrow\left(V^{1}\right)^{*} \otimes A \rightarrow \cdots \rightarrow\left(V^{n}\right)^{*} \otimes A \rightarrow \cdots .
$$

Item $(a)$ of the lemma is thus proven.

In order to prove item $(b)$, we have to write down a formula for the differential of complex (4.23). The resolution $R \rightarrow A$ gives an exact sequence

$$
0 \rightarrow J \hookrightarrow R^{0} \stackrel{\pi}{\rightarrow} A \rightarrow 0
$$


We shall regard an element $\tau \in\left(V^{j}\right)^{*}$ as a derivation of $R=S^{\bullet} V$. The differential, $D$, of $R$ can be written thus: $D=\sum f_{j} \partial_{j}+\xi$, where $\partial_{j} \in\left(V^{1}\right)^{*},\left\{f_{j}\right\}$ generate $J$, and $\xi \in F^{2} \operatorname{Der}(R)^{-1}$. It easily follows from the construction of the spectral sequence that if $\tau \in\left(V^{0}\right)^{*}$, then

$$
d^{1}(\tau \otimes a)=-a \pi\left(\tau\left(f_{j}\right)\right) \partial_{j} .
$$

It follows at once that $\operatorname{Ker}\left\{d^{1}:\left(V^{0}\right)^{*} \otimes A \rightarrow\left(V^{1}\right)^{*} \otimes A\right\}$ is precisely the algebra of derivations of $R^{0}$ that preserve the ideal $J$ modulo those derivations whose image is $J$, and this is $\operatorname{Der}(A)^{0}$ by definition.

\section{Quantization in the case of a Veronese ring}

5.1. Set-up. Consider the Veronese ring

$$
A_{N}=\mathbb{C}\left[x_{0}, \ldots, x_{N}\right] / Q, \quad Q=\left(x_{i} x_{j}-x_{i+1} x_{j-1}\right) .
$$

It is known that $\operatorname{Spec}\left(A_{N}\right)$ is the cone over the highest weight vector orbit in the projectivization of the $(N+1)$-dimensional representation of $s l_{2}$. Hence the canonical Lie algebra morphism

$$
s l_{2} \rightarrow \operatorname{Der}\left(A_{N}\right)
$$

An explicit formula for this morphism will appear in Section 5.2.3 below.

Being a cone, $\operatorname{Spec}\left(A_{N}\right)$ carries the Euler vector field $\sum_{j} x_{j} \partial_{j}$. This allows us to extend (5.2) to an action of $g l_{2}$ :

$$
g l_{2} \rightarrow \operatorname{Der}(A), \quad \text { where } \quad E_{11}+E_{22} \mapsto N \sum_{j} x_{j} \partial_{j}
$$

Remark. The normalizing factor of $N$ is not particularly important but can be justified by the geometry of the base affine space $S L_{2} / \mathcal{N}$.

As in Section 3.7.4, this gives a Courant algebroid morphism

$$
\mathcal{V}^{\text {poiss }}\left(g l_{2}\right) \rightarrow \mathcal{V}^{\text {poiss }}\left(A_{N}\right)^{0} \subset \mathcal{V}^{\text {poiss }}\left(A_{N}\right)^{\bullet}
$$

The following theorem, the main result of this paper, uses the concept of quantization of a Courant algebroid, see Definition 3.5, and the notion of quantization of a Courant algebroid map, see Section 3.7.4, (3.30), (3.31).

\section{Theorem 5.1.1.}

(a) The Courant algebroid $\mathcal{V}^{\text {poiss }}\left(A_{N}\right)^{\bullet}$ admits a unique quantization $\mathcal{V}\left(A_{N}\right)^{\bullet}$.

(b) Maps (5.3) and (4.21) can be quantized to the maps

$$
\begin{aligned}
& \mathcal{V}\left(g l_{2}\right)_{-N-2, N} \rightarrow \mathcal{V}\left(A_{N}\right)^{0}, \\
& \mathcal{U} \mathcal{L}(\mathcal{V} \text { ir })_{2} \rightarrow \mathcal{U}_{\text {alg }} \mathcal{V}\left(A_{N}\right)^{0},
\end{aligned}
$$

where the functors $\mathcal{U}$ and $\mathcal{U}_{\mathrm{alg}}$ are those defined in (2.8) and (3.23) resp.

The proof of the theorem is constructive, and an explicit construction of $\mathcal{V}\left(A_{N}\right)^{\bullet}$ will appear in Section 5.2.4 below. 


\subsection{Proof.}

5.2.1. Beginning of the proof: a reduction to the homological degree 0 . Suppose one quantization, $\mathcal{V}\left(A_{N}\right)^{\bullet}$, is given. We have the direct sum decomposition

$$
\mathcal{V}\left(A_{N}\right)^{\bullet}=\oplus_{n \in \mathbb{Z}} \mathcal{V}\left(A_{N}\right)^{n}
$$

where $\mathcal{V}\left(A_{N}\right)^{0} \subset \mathcal{V}\left(A_{N}\right)$ is a vertex subalgebroid. Filtration (3.12) in the present situation becomes

$$
A_{N} \oplus \Omega\left(A_{N}\right)^{0} \subset \mathcal{V}\left(A_{N}\right)^{\bullet}
$$

Since $A_{N} \oplus \Omega\left(A_{N}\right)^{0} \subset \mathcal{V}\left(A_{N}\right)^{0}, \mathcal{V}\left(A_{N}\right)^{0}$ is a quantization of $\mathcal{V}^{\text {poiss }}\left(A_{N}\right)^{0}$. This and the fact that $\Omega\left(A_{N}\right)^{\bullet}$ and $\operatorname{Der}\left(A_{N}\right)^{\bullet}$ are graded in opposite directions, cf. (4.8) and Lemma 4.5.1(a), imply a canonical vector space isomorphism

$$
\mathcal{V}\left(A_{N}\right)^{\bullet} \stackrel{\sim}{\longrightarrow} \mathcal{V}\left(A_{N}\right)^{0} \bigoplus\left(\oplus_{n<0} \operatorname{Der}\left(A_{N}\right)^{n}\right) \bigoplus\left(\oplus_{n>0} \Omega\left(A_{N}\right)^{n}\right)
$$

Now suppose that only $\mathcal{V}\left(A_{N}\right)^{0}$ is given.

Lemma. If $\mathcal{V}$ is a quantization of $\mathcal{V}^{\text {poiss }}\left(A_{N}\right)^{0}$, then there is a unique quantization, $\mathcal{V}\left(A_{N}\right)^{\bullet}$, of $\mathcal{V}^{\text {poiss }}\left(A_{N}\right)^{\bullet}$ such that $\mathcal{V}\left(A_{N}\right)^{0}=\mathcal{V}$.

Proof. ( $i$ ) Uniqueness. Pick a splitting (over $\mathbb{C}$ ) of the exact sequence of graded vector spaces, cf. (3.13),

$$
0 \rightarrow \Omega\left(A_{N}\right)^{0} \rightarrow \mathcal{V}\left(A_{N}\right)_{1}^{0} \rightarrow \operatorname{Der}\left(A_{N}\right)^{0} \rightarrow 0
$$

so as to identify $\mathcal{V}\left(A_{N}\right)_{1}$ with $\Omega\left(A_{N}\right) \oplus \operatorname{Der}\left(A_{N}\right)$ and obtain the projection $\pi: \mathcal{V}\left(A_{N}\right)_{1} \rightarrow \Omega\left(A_{N}\right)$ that is compatible with (5.7). It follows from Definition 3.5 that the only multiplications on $\mathcal{V}\left(A_{N}\right)$ that are not immediately determined by those on $\mathcal{V}^{\text {poiss }}\left(A_{N}\right)$ are the following components of $(0)$ and $(1)$ :

$$
\begin{aligned}
& \pi \circ\left(_{(0)}\right): \quad \operatorname{Der}\left(A_{N}\right)^{\bullet} \otimes \operatorname{Der}\left(A_{N}\right)^{\bullet} \rightarrow \Omega\left(A_{N}\right)^{\bullet}, \quad \xi \otimes \tau \mapsto \pi\left(\xi_{(0)} \tau\right), \\
& { }_{(1)}: \operatorname{Der}\left(A_{N}\right)^{\bullet} \otimes \operatorname{Der}\left(A_{N}\right)^{\bullet} \rightarrow A_{N}, \quad \xi \otimes \tau \mapsto \xi_{(1)} \tau .
\end{aligned}
$$

The homological degree of the l.h.s. of these is non-positive, see (5.7), of the r.h.s. is non-negative; therefore, the operations may be non-zero only if both $\xi, \tau \in \operatorname{Der}(A)^{0}$; the uniqueness follows.

(ii) To prove the existence, note that Definition 3.2 differs from Definition 3.3 in the following two respects only:

- the associativity of ${ }_{(-1)}$ in the former is replaced with quasi-associativity (2.5) in the latter;

- the requirements of the former that multiplications ${ }_{(n)}, n \geq 0$, be derivations of multiplication $(-1)$ and that multiplication $(-1)$ be commutative are simultaneously replaced with the Jacobi identity (2.4) with $m$ or $n$ equal to -1 .

Upon choosing a splitting as at the beginning of the proof, in each of the cases the identities of Definition 2.2 exhibit quantum corrections, i.e., the terms that measure the failure of a quantum object to be a classical one. It is easy to notice, by inspection, that in our situation the quantum corrections may be non-zero only if all the terms involved belong to $\mathcal{V}=\mathcal{V}\left(A_{N}\right)^{0}$.

One such example is provided by formula (3.16), where the failure of multiplication $(-1)$ to be associative is measured by $-\tau(g) d f-\tau(f) d g$; both the summands vanish unless $\tau \in \mathcal{V}, f, g$ being in $\mathcal{V}$ automatically. 
Another example deals with the commutativity of ${ }_{(-1)}$. Let $f \in A_{N}, \tau \in \operatorname{Der}\left(A_{N}\right)^{\bullet}$; then $(2.4)$ reads

$$
\left[\tau_{(-1)}, f_{(-1)}\right]=\tau(f)_{(-2)},
$$

which is 0 unless $\tau \in \mathcal{V}$. We leave it to the untiring reader to check the validity of all the remaining requirements of Definition 3.3.

In order to prove Theorem 5.1.1, it remains to quantize $\mathcal{V}^{\text {poiss }}\left(A_{N}\right)^{0}$. We shall do this in a somewhat roundabout manner.

5.2.2. Localization. To return to the hypothetical vertex algebroid $\mathcal{V}\left(A_{N}\right)^{\bullet}$. By virtue of Section 5.2.1, it is enough to consider $\mathcal{V}\left(A_{N}\right) \stackrel{\text { def }}{=} \mathcal{V}\left(A_{N}\right)^{0}$. Since $C=\operatorname{Spec}\left(A_{N}\right)$ is affine, we can localize $\mathcal{V}\left(A_{N}\right)$, see (3.25) in Section 3.7.1, so as to get a sheaf $\mathcal{V}_{C} \in \mathcal{V}$ ert $_{C}$. Let $\check{C}$ be $C \backslash\{0\}$ and $\mathcal{V}_{\breve{C}}$ the restriction of $\mathcal{V}_{C}$ to $\check{C}$. Apparently, $\mathcal{V}_{\breve{C}} \in \mathcal{V}_{\operatorname{ert}_{\check{C}}}$ and, the manifold $\check{C}$ being smooth, our strategy will be to use the classification of the objects of $\mathcal{V}_{\check{C}}$, Section 3.7.3, so as to identify those vertex algebroids over $\check{C}$ that may have come from $C$ as above.

We begin by realizing $\check{C}$ as a quotient of a manifold w.r.t. a finite group action. Consider the action

$$
\mathbb{Z}_{N} \times \mathbb{C}^{2} \rightarrow \mathbb{C}^{2}, \quad \bar{m}\left(y_{1}, y_{2}\right)=\left(\exp (2 \pi \sqrt{-1} m / N) y_{1}, \exp (2 \pi \sqrt{-1} m / N) y_{2}\right) .
$$

The map

$$
A_{N} \rightarrow \mathbb{C}\left[y_{1}, y_{2}\right]^{\mathbb{Z}_{N}}, \quad x_{j} \mapsto y_{1}^{N-j} y_{2}^{j}
$$

is an isomorphism; hence isomorphisms

$$
\mathbb{C}^{2} / \mathbb{Z}_{N} \stackrel{\sim}{\longrightarrow} C, \quad\left(\mathbb{C}^{2} \backslash 0\right) / \mathbb{Z}_{N} \stackrel{\sim}{\longrightarrow} \check{C} .
$$

There arises a projection

$$
p: \quad \mathbb{C}^{2} \backslash 0 \rightarrow \check{C}
$$

and a faithful functor

$$
p^{*}: \operatorname{Vert}_{\check{C}} \rightarrow \mathcal{V} \operatorname{ert}_{\mathbb{C}^{2} \backslash 0}
$$

It is an equivalence of categories

$$
p^{*}: \operatorname{Vert}_{\check{C}} \rightarrow \mathcal{V} \operatorname{vrt}_{\mathbb{C}^{2} \backslash 0}^{\mathbb{Z}_{N}}
$$

where $\mathcal{V}$ ert $\mathbb{C}^{2} \backslash 0$ is the full subcategory of $\mathbb{Z}_{N}$-equivariant vertex algebroids; the inverse functor is that of $\mathbb{Z}_{N^{\text {-invariants: }}} \mathcal{V}_{\mathbb{C}^{2} \backslash 0} \mapsto \mathcal{V}_{\mathbb{C}^{2} \backslash 0}^{\mathbb{Z}_{0}}$; cf. Section 3.7.1, (3.26), (3.27).

The objects of the category $\mathcal{V} \operatorname{rt}_{\mathbb{C}^{2} \backslash 0}$ were classified in Section 3.7.3 to the effect that there is a 1-1 correspondence between isomorphism classes of vertex algebroids and linear combinations $\omega=\sum_{a, b>0} k_{a b} \omega_{a b}$, where $\omega_{a b}$ is the 2 -form $d y_{1} \wedge d y_{2} / y_{1}^{a} y_{2}^{b}$. It follows from the construction that the vertex algebroid, $\mathcal{V}_{\mathbb{C}^{2} \backslash 0, \omega}$ is $\mathbb{Z}_{N^{-}}$equivariant if and only if $\omega$ is, hence if and only if

$$
\omega=\sum_{a, b>0, N} k_{a b} \omega_{a b} .
$$

It is from this list that we have to select. 
5.2.3. Conclusion of the proof. By definition, our hypothetical sheaf $\mathcal{V}_{C}$ must fit, for some $\omega$, in the following commutative diagram:

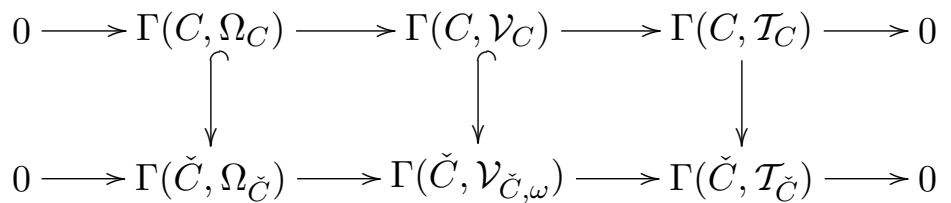

Note that the vertical arrows are all the restriction (from $C$ to $\check{C}$ ) maps. Furthermore, the rightmost vertical arrow is an equality. To see this, note that $\Gamma\left(\check{C}, \mathcal{T}_{\check{C}}\right)=\Gamma\left(\mathbb{C}^{2} \backslash 0, \mathcal{T}_{\mathbb{C}^{2} \backslash 0}\right)^{\mathbb{Z}_{N}}$. The latter is generated, over functions, by the tautological action of $g l_{2}$, see Section 3.7.4, formulas (3.32), (3.33). (Indeed, an element of $\Gamma\left(\mathbb{C}^{2} \backslash 0, \mathcal{T}_{\mathbb{C}^{2} \backslash 0}\right)^{\mathbb{Z}_{N}}$ is a linear combination of $f\left(y_{1}, y_{2}\right) \partial_{1}$ and $g\left(y_{1}, y_{2}\right) \partial_{2}$, where $N$ divides $\operatorname{deg}(f)-1$ and $\operatorname{deg}(g)-1$. This implies that $f\left(y_{1}, y_{2}\right) \partial_{1}$ is proportional to either $\rho\left(E_{11}\right)$ or to $\rho\left(E_{21}\right)$ and $g\left(y_{1}, y_{2}\right) \partial_{2}$ is proportional to either $\rho\left(E_{12}\right)$ or to $\rho\left(E_{22}\right)$, see (3.33).) Therefore, so is the former. But this action is precisely the action of $g l_{2}$ on $C=\operatorname{Spec}\left(A_{N}\right)$ described somewhat implicitly in $(5.2)$, hence the equality $\Gamma\left(C, \mathcal{T}_{C}\right)=\Gamma\left(\check{C}, \mathcal{T}_{\breve{C}}\right)$.

Contrary to this, the leftmost vertical arrow is not an equality; e.g. it is easy to check that

$$
y_{1}^{r} y_{2}^{N-r-1} d y_{1} \in \Gamma\left(\check{C}, \Omega_{\check{C}}\right) \quad \text { but } \quad y_{1}^{r} y_{2}^{N-r-1} d y_{1} \notin \Gamma\left(C, \Omega_{C}\right) \quad \text { if } \quad 0 \leq r \leq N-2 .
$$

This simple remark is the reason why the quantization of $\mathcal{V}^{\text {poiss }}\left(A_{N}\right)$ is unique.

Now, the upper row of (5.15) is exact by virtue of Definition 3.5. This and the fact that the rightmost arrow is an equality imply that the lower row must also be exact, at least on the right. By virtue of Corollary 3.7.6,

$$
\omega=k \omega_{11} \text {. }
$$

Now our task is to determine $k$.

Define $\mathcal{W}$ to be the vertex subalgebroid of $\Gamma\left(\check{C}, \mathcal{V}_{\check{C}, k \omega_{11}}\right)$ generated by $A_{N}=\Gamma\left(\check{C}, \mathcal{O}_{\check{C}}\right)$ and $\hat{\rho}\left(\mathcal{V}\left(g l_{2}\right)_{-k-1, k-1}\right)$, where $\hat{\rho}$ is the one from Lemma 3.7.5.

Since $\Gamma\left(C, \mathcal{T}_{C}\right)$ is generated by $\rho\left(g l_{2}\right)$ over $A_{N}, \mathcal{W}=\Gamma\left(C, \mathcal{V}_{C}\right)$. It is clear that $\mathcal{W}=A_{N} \oplus$ $\Gamma\left(C, \Omega_{C}\right)+A_{N(-1)} \hat{\rho} \mathcal{V}^{\mathrm{ch}}\left(g l_{2}\right)_{-k-1, k-1}$ and were the elements $\rho\left(E_{i j}\right)$ independent over $A_{N}$, we would be done: $\mathcal{W}$ would be the sought after quantization for any $k$. (In fact, were that true, we could equivalently define $\mathcal{V}\left(A_{N}\right)$ to be the push-out $A_{N} \otimes \mathcal{V}\left(g l_{2}\right)_{-k-1, k-1}$, see (3.28).) But they are not, and the problem with this is that an element of $A_{N(-1)} \hat{\rho} \mathcal{V}^{\text {ch }}\left(g l_{2}\right)_{-k-1, k-1}$ may belong to $\Gamma\left(\check{C}, \Omega_{\check{C}}\right)$ and not to $\Gamma\left(C, \Omega_{C}\right)$, cf. (5.16).

In fact, $A_{N}$ is a quadratic algebra, see $(5.1)$, and $\Gamma\left(C, \mathcal{I}_{C}\right)$ is a quadratic $A_{N}$-module generated by $\left\{E_{i j}, 1 \leq i, j \leq 2\right\}$. The relations, in terms of $y_{1}, y_{2}$, are

$$
y_{1}^{a} y_{2}^{b} y_{k} \hat{\rho}\left(E_{i j}\right)-y_{1}^{a} y_{2}^{b} y_{i} \hat{\rho}\left(E_{k j}\right)=0 \quad \text { for all } a+b+1=N,
$$

as it easily follows from (3.33).

Our task then is to ensure that

$$
\left(y_{1}^{a} y_{2}^{b} y_{k}\right)_{(-1)} \hat{\rho}\left(E_{i j}\right)-\left(y_{1}^{a} y_{2}^{b} y_{i}\right)_{(-1)} \hat{\rho}\left(E_{k j}\right) \in \Gamma\left(C, \Omega_{C}\right) \quad \text { for all } \quad a+b+1=N .
$$

Let us consider for the sake of definiteness the case of $i=1, k=j=2$. We have to compute the following section of $\Gamma\left(\check{C}, \mathcal{V}_{\check{C}, k \omega_{11}}(\check{C})\right)$ :

$$
\left(y_{1}^{r} y_{2}^{N-r}\right)_{(-1)} \hat{\rho}\left(E_{12}\right)-\left(y_{1}^{r+1} y_{2}^{N-r-1}\right)_{(-1)} \hat{\rho}\left(E_{22}\right) \quad \text { for all } \quad 0 \leq r<N .
$$

Formulas (3.34)-(3.37) allow us to re-write (5.20) as follows

$$
\left(y_{1}^{r} y_{2}^{N-r}\right)_{(-1)}\left(y_{1} \partial_{2}\right)-\left(y_{1}^{r+1} y_{2}^{N-r-1}\right)_{(-1)}\left(y_{2} \partial_{y_{2}}+\frac{k T\left(y_{1}\right)}{y_{1}}\right) .
$$


A little thought (or formula (3.16)) will show that the first term yields

$$
y_{1}^{r+1} y_{2}^{N-r} \partial_{2}-r(N-r) y_{1}^{r} y_{2}^{N-r-1} T\left(y_{1}\right)-(N-r)(N-r-1) y_{1}^{r+1} y_{2}^{N-r-2} T\left(y_{2}\right),
$$

where it is understood that

$$
y_{1}^{r+1} y_{2}^{N-r} \partial_{2} \stackrel{\text { def }}{=}\left(y_{1(-1)}\left(y_{1(-1)}\left(\cdots\left(y_{1(-1)}\left(y_{2(-1)}\left(\cdots\left(y_{2(-1)} \partial_{2}\right) \cdots\right)\right)\right)\right)\right)\right) \text {. }
$$

The second one will likewise give

$$
\begin{aligned}
& -y_{1}^{r+1} y_{2}^{N-r} \partial_{2}+(N-r-1)(r+1) y_{1}^{r} y_{2}^{N-r-1} T\left(y_{1}\right) \\
& \quad+(N-r-1)(N-r-2) y_{1}^{r+1} y_{2}^{N-r-2} T\left(y_{2}\right)-k y_{1}^{r} y_{2}^{N-r-1} T\left(y_{1}\right) .
\end{aligned}
$$

Adding one to another makes expression (5.20) into

$$
(N-1-2 r-k) y_{1}^{r} y_{2}^{N-r-1} T\left(y_{1}\right)+(-2 N+2 r+2) y_{1}^{r+1} y_{2}^{N-r-2} T\left(y_{2}\right) .
$$

The latter equals the total derivative

$$
-2 T\left(y_{1}^{r+1} y_{2}^{N-r-1}\right)=-2 T\left(x_{N-r-1}\right),
$$

and is therefore an element of $\Gamma\left(C, \Omega_{C}\right)$, precisely when $k=N+1$.

The case where $i=j=1, k=2$ works out similarly and gives the same answer $k=N+1$. This concludes the proof of item (a) of Theorem 5.1.1.

As to item $(b),(5.5)$ follows from the $k=N+1$ case of Lemma 3.7.5, the assertion that has been instrumental for the proof anyway, and (5.6) follows from (3.40).

Corollary 5.2.4. The unique quantization $\mathcal{V}\left(A_{N}\right)=\mathcal{V}\left(A_{N}\right)^{0} \oplus \operatorname{Der}\left(A_{N}\right)^{\bullet} \oplus \Omega\left(A_{N}\right)^{\bullet}$, where $\mathcal{V}\left(A_{N}\right)^{0}$ is the vertex subalgebroid of $\Gamma\left(\check{C}, \mathcal{V}_{\check{C},(N+1) \omega_{11}}\right)$ generated by $A_{N}=\Gamma\left(\check{C}, \mathcal{O}_{\check{C}}\right)$ and $\hat{\rho}\left(\mathcal{V}\left(g l_{2}\right)_{-N-2, N}\right), \hat{\rho}$ being the one from Lemma 3.7.5.

5.3. Higher dimensional Veronese embeddings. Regard $\mathbb{C}^{n}$ as the tautological representation of $g l_{n}$ and let $V=\left(\mathbb{C}^{n}\right)^{*}$. Let

$$
\iota_{N}: \quad \mathbb{P}(V) \rightarrow \mathbb{P}\left(S^{N} V\right), \quad l \mapsto l^{\otimes N}
$$

be the classical Veronese embedding. By $A_{n N}$ let us denote the homogeneous coordinate ring of $\iota_{N}(\mathbb{P}(V))$. It is clear that if $n=2$, then $A_{n N}$ is the algebra $A_{N}$ we dealt with above. It is now natural to ask if $\mathcal{V}^{\text {poiss }}\left(A_{n N}\right)$ affords a quantization. The result is a bit disheartening.

Theorem 5.3.1. The vertex Poisson algebroid $\mathcal{V}^{\text {poiss }}\left(A_{n N}\right)$ cannot be quantized if $N>1$ and $n>2$.

Proof. Consider the action

$$
\mathbb{Z}_{N} \times V \rightarrow V, \quad(\bar{m}, v) \mapsto \exp (2 \pi \sqrt{-1} m / N) v .
$$

Analogously to (5.9), (5.10), we obtain isomorphisms

$$
V / \mathbb{Z}_{N} \stackrel{\sim}{\longrightarrow} \operatorname{Spec}\left(A_{n N}\right), \quad(V \backslash 0) / \mathbb{Z}_{N} \stackrel{\sim}{\longrightarrow} \operatorname{Spec}\left(A_{n N}\right) \backslash 0 .
$$

Thus we are led to the question, "How many vertex algebroids are there on $(V \backslash 0) / \mathbb{Z}_{N}$ ?" That such vertex algebroids exist is obvious because $\mathcal{V}_{V \backslash 0}^{\mathbb{Z}_{N}}$ is one; here $\mathcal{V}_{V \backslash 0}$ is the pull-back of the standard $\mathcal{V}_{V}$, cf. Section 3.7.2, on $V \backslash 0$. Note that if $x_{1}, \ldots, x_{n}$ is a basis of $\mathbb{C}^{n}$ - remember that we think of $\mathbb{C}^{n}$ as the space of linear functions on $V$ - then the assignment

$$
\hat{\rho}: \quad E_{i j} \mapsto x_{i(-1)} \partial_{j}
$$

defines a vertex algebroid morphism

$$
\hat{\rho}: \quad \mathcal{V}\left(g l_{n}\right)_{-1,-1} \rightarrow \Gamma\left((V \backslash 0) / \mathbb{Z}_{N}, \mathcal{V}_{V \backslash 0}^{\mathbb{Z}_{N}}\right) .
$$


Lemma 5.3.2. The manifolds $V \backslash 0,(V \backslash 0) / \mathbb{Z}_{N}$ carry a unique up to isomorphism sheaf of vertex algebroids. It is isomorphic to $\mathcal{V}_{V \backslash 0}$ in the former case and to $\mathcal{V}_{V \backslash 0}^{\mathbb{Z}_{N}}$ in the latter case.

Proof of Lemma 5.3.2. By virtue of (3.22) and (3.26), it suffices to show that

$$
H^{1}\left(V \backslash 0, \Omega_{V \backslash 0}^{2} \rightarrow \Omega_{V \backslash 0}^{3, \mathrm{cl}}\right)=0 .
$$

Converging to the hypercohomology $H^{*}\left(V \backslash 0, \Omega_{V \backslash 0}^{2} \rightarrow \Omega_{V \backslash 0}^{3, \mathrm{cl}}\right)$ is a standard spectral sequence with

$$
\begin{aligned}
& E_{00}^{1}=H^{0}\left(V \backslash 0, \Omega_{V \backslash 0}^{2}\right), \\
& E_{01}^{1} \oplus E_{10}^{1}=H^{1}\left(V \backslash 0, \Omega_{V \backslash 0}^{2}\right) \oplus H^{0}\left(V \backslash 0, \Omega_{V \backslash 0}^{3, \mathrm{cl}}\right) .
\end{aligned}
$$

The next differential is

$$
d^{2}=d_{D R}: \quad H^{0}\left(V \backslash 0, \Omega_{V \backslash 0}^{2}\right) \rightarrow H^{0}\left(V \backslash 0, \Omega_{V \backslash 0}^{3, \mathrm{cl}}\right),
$$

and it is clear that it is surjective. Finally, if $n>2$, then $H^{1}\left(V \backslash 0, \Omega_{V \backslash 0}^{2}\right)=0$; this concludes the proof of Lemma 5.3.2.

It is clear now why $\mathcal{V}^{\text {poiss }}\left(A_{n N}\right)$ cannot be quantized if $n>2$ : it is because Lemma 5.3.2 has left us no room for manoeuvre that was helpfully provided by the analysis of Section 3.7.3 in the $n=2$ case. Indeed, one can now repeat the entire argument of Sections 5.2.1-5.2.3 only to find out that an obvious analogue of (5.19) is false. Here are some details:

According to Lemma 5.3.2, a quantization of $\mathcal{V}^{\text {poiss }}\left(A_{n N}\right)$, if existed, upon localizing to $(V \backslash 0) / \mathbb{Z}_{N}$ would give $\mathcal{V}_{V \backslash 0}^{\mathbb{Z}_{N}}$. Hence any such quantization can be equal only to the vertex subalgebroid of $\Gamma\left(V \backslash 0, \mathcal{V}_{V \backslash 0}\right)^{\mathbb{Z}_{N}}$ generated by $A_{n N}$ and $\hat{\rho}\left(\mathcal{V}\left(g l_{n}\right)_{-1,-1}\right.$, see (5.24-5.25). But this subalgebroid necessarily contains elements from $\Gamma\left((V \backslash 0) / \mathbb{Z}_{N}, \Omega_{(V \backslash 0) / \mathbb{Z}_{N}}\right) \backslash \Omega\left(A_{n N}\right)$. Indeed, a computation analogous to the one performed at the end of Section 5.2.3 shows that if $n \geq 3$, then

$$
\begin{aligned}
& \left(x_{3} x_{2}^{N-1}\right)_{(-1)}\left(x_{1(-1)} \partial_{1}\right)-\left(x_{3} x_{2}^{N-2} x_{1}\right)_{(-1)}\left(x_{2(-1)} \partial_{1}\right) \\
& \left.\quad=\left(T\left(x_{3} x_{2}^{N-2}\right)\right)_{(-1)} x_{2} \in \Gamma\left((V \backslash 0) / \mathbb{Z}_{N}, \Omega_{(V \backslash 0) / \mathbb{Z}_{N}}\right)\right) \backslash \Omega\left(A_{n N}\right) .
\end{aligned}
$$

This concludes the proof of Theorem 5.3.1.

\section{Chiral Hamiltonian reduction interpretation}

We will now interpret some of the constructions above in the language of semi-infinite cohomology. Our exposition will be brief and almost no proofs will be given. In some respects, the material of this section is but an afterword to [16].

Since we will be mostly concerned with smooth varieties, we will find it convenient to work not with vertex algebroids, such as $\mathcal{V}(\mathfrak{g})_{k}, \mathcal{V}_{X}$, but with the corresponding vertex algebras or CDO-s, such as $\mathcal{U}_{\text {alg }} \mathcal{V}(\mathfrak{g})_{k}=\mathcal{U} \mathcal{L}(\mathfrak{g})_{k}, D_{X}^{\text {ch }}=\mathcal{U}_{\text {alg }} \mathcal{V}_{X}$

6.1. Semi-infinite cohomology. Let $\mathbb{V}$ be a vertex algebra, $\mathfrak{g}$ a finite dimensional Lie algebra, $(\cdot, \cdot)$ an invariant bilinear form on $\mathfrak{g}$, and $\rho$ a vertex algebra morphism

$$
\rho: \quad \mathcal{U}_{\text {alg }} \mathcal{V}(\mathfrak{g})_{(\cdot, \cdot)} \rightarrow \mathbb{V}
$$

see Example 2.5, especially (2.12). 
Introduce the Clifford vertex algebra built on $\Pi\left(\mathfrak{g} \oplus \mathfrak{g}^{*}\right)$, $\Pi$ being the parity change functor. This vertex algebra is nothing but the space of global sections of the standard CDO on superspace $\Pi\left(\mathfrak{g} \oplus \mathfrak{g}^{*}\right)$, see Section 3.7.2 for a discussion of a purely even analogue. Denote this vertex algebra by $D^{\operatorname{ch}}\left(\Pi\left(\mathfrak{g} \oplus \mathfrak{g}^{*}\right)\right)$.

By definition, if we let $\left\{x_{i}\right\}$ be a basis of $\mathfrak{g},\left\{\phi_{i}\right\}$ the corresponding basis of $\Pi(\mathfrak{g}),\left\{\phi_{i}^{*}\right\}$ the dual basis of $\Pi\left(\mathfrak{g}^{*}\right)$, then $D^{\text {ch }}\left(\Pi\left(\mathfrak{g} \oplus \mathfrak{g}^{*}\right)\right)$ is the vertex algebra generated by the vector space $\Pi\left(\mathfrak{g} \oplus \mathfrak{g}^{*}\right)$ and relations

$$
\begin{aligned}
& \left(\phi_{i}^{*}\right)_{(0)} \phi_{j}=\left(\phi_{i}\right)_{(0)} \phi_{j}^{*}=\delta_{i j} \mathbf{1}, \\
& \left(\phi_{i}^{*}\right)_{(n+1)} \phi_{j}=\left(\phi_{i}\right)_{(n+1)} \phi_{j}^{*}=\left(\phi_{i}^{*}\right)_{(n)} \phi_{j}^{*}=\left(\phi_{i}\right)_{(n)} \phi_{j}^{*}=0 \quad \text { if } \quad n \geq 0 .
\end{aligned}
$$

There arises the vertex algebra $\mathbb{V} \otimes D^{\mathrm{ch}}\left(\Pi\left(\mathfrak{g} \oplus \mathfrak{g}^{*}\right)\right)$.

If $\left\{c_{i j}^{k}\right\}$ are the structure constants of $\mathfrak{g}$ relative to $\left\{x_{i}\right\}$, that is to say, if $\left[x_{i}, x_{j}\right]=\sum_{k} c_{i j}^{k} x_{k}$, then following [13] one considers the element

$$
d^{\infty / 2}=\sum_{k} \rho\left(x_{k}\right)_{(-1)} \phi_{k}^{*}-\frac{1}{2} \sum_{i, j, k} c_{i j}^{k} \phi_{k(-1)}\left(\phi_{i(-1)}^{*} \phi_{j}^{*}\right) \in \mathbb{V} \otimes D^{\mathrm{ch}}\left(\Pi\left(\mathfrak{g} \oplus \mathfrak{g}^{*}\right)\right) .
$$

A direct computation shows that

$$
\left(d^{\infty / 2}\right)_{(0)} d^{\infty / 2}=0 \quad \text { if } \quad(\cdot, \cdot)=-K(\cdot, \cdot)
$$

where $K(\cdot, \cdot)$ is the Killing form on $\mathfrak{g}: K(a, b)=\operatorname{tr}\left(\operatorname{ad}_{a} \cdot \operatorname{ad}_{b}\right)$.

If condition (6.2) is satisfied, then we obtain a DGVA $C^{\infty / 2}(L \mathfrak{g} ; \mathbb{V}) \stackrel{\text { def }}{=}\left(\mathbb{V} \otimes D^{\text {ch }}\left(\Pi\left(\mathfrak{g} \oplus \mathfrak{g}^{*}\right)\right)\right.$, $\left.d_{(0)}^{\infty / 2}\right)$. The cohomology vertex algebra $H^{\infty / 2}(L \mathfrak{g} ; \mathbb{V})$ is due to Feigin [13] and well known as either semi-infinite or BRST cohomology of the loop algebra $L \mathfrak{g}$ with coefficients in $\mathbb{V}$. If one chooses to think of $\mathbb{V}$ as an algebra of ('chiral') functions on a symplectic manifold with $\mathfrak{g}$ structure, then $H^{\infty / 2}(L \mathfrak{g}, \mathbb{V})$ is to be thought of as an algebra of functions on the symplectic quotient $M / / \mathfrak{g}$, hence the title of this section.

One similarly defines the relative version $H^{\infty / 2}(L \mathfrak{g}, \mathfrak{g} ; \mathbb{V})$, see [16] for some details; the condition (6.2) remains the same in this case.

6.2. The $s l_{2}$ case. Let us return to the set-up of Section 5.2.2, where we had the Veronese cone $C=\operatorname{Spec}\left(A_{N}\right), \check{C}=C \backslash 0$, and consider $\mathcal{L}_{N}$, the degree $N$ line bundle over $\mathbb{P}^{1}$, and $\check{\mathcal{L}}_{N}=\mathcal{L}_{N} \backslash\{$ the zero section $\}$. We obtain the commutative square

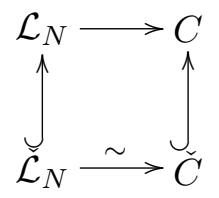

where the upper horizontal map is a surjective birational isomorphism, a blow-up of the vertex of the cone. We have seen that $\check{C}$ carries a family of CDO-s, $D_{\check{C}, \omega}^{\text {ch }}, \omega \in H^{1}\left(\check{C}, \Omega_{\check{C}}^{2}\right)$. Denote the coorresponding family of CDO-s on $\check{\mathcal{L}}_{N}$ by $D_{\check{\mathcal{L}}_{N}, \omega}^{\text {ch }}$. Theorem 5.1 .1 says that $D_{\check{C}, \omega}^{\text {ch }}$ is a pull-back of a CDO on $C$ iff $\omega=(N-1) \omega_{11}$, in which case it contains $\mathcal{V}\left(s l_{2}\right)_{-N-2}$.

Now a question arises, "For what, if any, $\omega$ is $D_{\tilde{\mathcal{L}}_{N}, \omega}^{\text {ch }}$ a pull-back of a CDO from $\mathcal{L}_{N}$ ?" The existence of such $\omega$ depends on the vanishing of the characteristic class $\operatorname{ch}_{2}\left(\mathcal{L}_{N}\right),(3.21)$. A simple way to prove the vanishing result, and to compute a possible $\omega$, is provided by the semi-infinite cohomology. 
One has the base affine space $\mathbb{C}^{2} \backslash 0$, the principal $N$-bundle $p: S L_{2} \rightarrow \mathbb{C}^{2} \backslash 0$, where $N$ is the subgroup of upper-triangular matrices and a family of CDO-s, $\mathcal{D}_{S L_{2},(\cdot, \cdot)}$ on $S L_{2}$, over $S L_{2}$. This family enjoys $[1,16]$ the 2 vertex algebra embeddings

$$
\begin{aligned}
& \mathcal{U}_{\text {alg }} \mathcal{V}(\mathfrak{g})_{(\cdot, \cdot)} \stackrel{\rho_{l}}{\longrightarrow} \Gamma\left(S L_{2}, D_{S L_{2},(\cdot, \cdot)}^{\text {ch }}\right) \stackrel{\rho_{r}}{\leftarrow} \mathcal{U}_{\text {alg }} \mathcal{V}(\mathfrak{g})_{-(\cdot, \cdot)-K(\cdot, \cdot)}, \\
& \text { s.t. } \quad \rho_{l}(a)_{(n)} \rho_{r}(b)=0 \quad \text { if } \quad n \geq 0 .
\end{aligned}
$$

In this case condition (6.2) is satisfied for all forms $(\cdot, \cdot)$. Therefore, for any $U \subset S L_{2} / N$, there arises a vertex algebra $H^{\infty}\left(L \mathfrak{n}, \Gamma\left(p^{-1}(U), D_{S L_{2}, k}^{\text {ch }}\right)\right)$, where we use $\rho_{r}$ in place of $\rho$, see $(6.1)$. Denote by $H^{\infty / 2}\left(L \mathfrak{n}, D_{S L_{2},(\cdot, \cdot)}^{\text {ch }}\right)$ the sheaf associated with the presheaf $U \mapsto H^{\infty}\left(L \mathfrak{n}, \Gamma\left(p^{-1}(U)\right.\right.$, $\left.\mathcal{D}_{S L_{2},(\cdot, \cdot)}\right)$. It was noted in [16] that this sheaf is a CDO, which re-proves the obvious fact that $\operatorname{ch}_{2}\left(\mathbb{C}^{2} \backslash 0\right)=0$.

Note that the left one of the embeddings (6.4) survives the passage to the cohomology. The right one does not, not entirely at least, but the embedding of the torus part does, albeit with a shifted central charge. We obtain

$$
\mathcal{U}_{\text {alg }} \mathcal{V}(\mathfrak{g})_{(\cdot, \cdot)} \stackrel{\rho_{l}}{\rightarrow} \Gamma\left(\mathbb{C}^{2} \backslash 0, H^{\infty / 2}\left(L \mathfrak{n}, D_{S L_{2},(\cdot,)}^{\text {ch }}\right) \stackrel{\rho_{r}}{\leftarrow} \mathcal{U}_{\text {alg }} \mathcal{V}(\mathfrak{t})_{-\left.(\cdot, \cdot)\right|_{\mathfrak{t}}-1 /\left.2 K(\cdot, \cdot)\right|_{\mathfrak{t}}}\right.
$$

so that $\rho_{l}(a)_{(n)} \rho_{l}(b)=0$ if $n \geq 0$; here $\left.(\cdot, \cdot)\right|_{\mathfrak{t}}$ and $\left.K(\cdot, \cdot)\right|_{\mathfrak{t}}$ stand for the restrictions of the corresponding forms to $t$.

Altogether, the 2 embeddings provide a vertex algebra embedding of $\mathcal{U}_{\text {alg }} \mathcal{V}\left(g l_{2}\right)_{k_{1}, k_{2}}$ with appropriate central charges $k_{1}$ and $k_{2}$. In fact, if we let $(a, b)=k \operatorname{tr}(a \cdot b)$ as we did in Section 3.7.4, then we obtain that $k_{1}=k, k_{2}=-k-2$ and a diagram, cf. Lemma 3.7.5 and (3.38).

$$
\mathcal{U}_{\text {alg }} \mathcal{V}(\mathfrak{g})_{k} \hookrightarrow \Gamma\left(\mathbb{C}^{2} \backslash 0, H^{\infty / 2}\left(L \mathfrak{n}, D_{S L_{2},(\cdot, \cdot)}^{\text {ch }}\right) \stackrel{\sim}{\longrightarrow} \Gamma\left(\mathbb{C}^{2} \backslash 0, D_{\mathbb{C}^{2} \backslash 0,-(k+1) \omega_{11}}^{\text {ch }}\right) .\right.
$$

Lemma 3.7.5 shows that the chiral hamiltonian reduction technology reproduces precisely those CDO-s on the punctured plane that carry an affine Lie algebra action.

In order to try and define a $\mathrm{CDO}$ on $\mathcal{L}_{N}$, we represent the latter as

$$
\mathcal{L}_{N}=\left(\mathbb{C}^{2} \backslash 0\right) \times_{\mathbb{C}^{*}} \mathbb{C}_{N},
$$

where $\mathbb{C}_{N}$ is the character $\mathbb{C}^{*} \ni z \mapsto z^{N}$. This suggests to define a CDO on $\mathcal{L}_{N}$ as the chiral hamiltonian reduction of $D_{\mathbb{C}^{2} \backslash 0,-(k+1) \omega_{11}}^{\text {ch }} \otimes D_{\mathbb{C}}^{\text {ch }}$ using embedding (6.5) twisted by action (6.7); in practice that means that if $h$ is the standard generator of $\mathfrak{t}$, then one has to replace $\rho_{r}(h)$ in (6.5) with $\rho_{r}(h)+N y_{(-1)} \partial_{y}$, where $y$ is a coordinate on $\mathbb{C}_{N}$. Two things are to be kept in mind: first, since the topology of $\mathbb{C}^{*}$ is non-trivial [16], one has to use the relative version of the semi-infinite cohomology; second, and most important, condition (6.2) is not automatically satisfied. In fact, (6.2) is equivalent to

$$
\left(\rho_{r}(h)+N y_{(-1)} \partial_{y}\right)_{(1)}\left(\rho_{r}(h)+N y_{(-1)} \partial_{y}\right)=0,
$$

which gives

$$
(\cdot, \cdot)=-\left(\frac{N^{2}}{8}+\frac{1}{2}\right) K(\cdot, \cdot) .
$$

It follows that $\mathcal{H}^{\infty / 2}\left(L \mathfrak{t}, \mathfrak{t} ; D_{\mathbb{C}^{2} \backslash 0,-(k+1) \omega_{11}}^{\text {ch }} \otimes D_{\mathbb{C}}^{\text {ch }}\right)$ is well defined and gives a CDO on $\mathcal{L}_{N}$ precisely if (6.9) holds.

To conclude,

(1) the manifold $\check{\mathcal{L}}_{N}$ carries a 1-parameter family of CDO-s, $D_{\mathbb{C}^{2} \backslash 0,-(k+1) \omega_{11}}^{\text {ch }}$, with $\mathcal{U}_{\text {alg }} \mathcal{V}\left(s l_{2}\right)_{(\cdot, \cdot)}$-structure, see Section 3.7.4; 
(2) the condition that a $\mathrm{CDO}$ on $\check{C}$ extends to one on $C$ picks a unique $(\cdot, \cdot)$; the latter depends on $N$ linearly, see Theorem 5.1.1;

(3) the family $D_{\mathbb{C}^{2} \backslash 0,-(k+1) \omega_{11}}^{\text {ch }}$ contains at least one representative that extends to a CDO on $\mathcal{L}_{N}$; the condition that a $\mathrm{CDO}$ on $\check{\mathcal{L}}_{N}$ extends to $\mathcal{L}_{N}$ and affords a realization via the chiral Hamiltonian reduction picks a unique $(\cdot, \cdot)$; the latter depends on $N$ quadratically, (6.9).

In fact, there is a third way to fix a $(\cdot, \cdot)$. This one amounts to carrying a regularization procedure à la Lambert, used in [4] in a similar but different situation, and gives another quadratic dependence on $N$. The importance of this approach is yet to be worked out.

6.3. Higher rank generalization. Let $G$ be a simple complex Lie group, $P \subset G$ a parabolic subgroup, $R \subset P$ the unipotent radical of $R, M=P / R$. Let us make the following assumption

$$
M=M_{0} \times M_{1}, \quad \text { where } \quad M_{0} \stackrel{\sim}{\longrightarrow} \mathbb{C}^{*}, \quad M_{1} \text { is simple. }
$$

Let $Q \subset G$ be the extension of $M_{1}$ by $R$. Thus we obtain a $\mathbb{C}^{*}$-bundle

$$
G / Q \rightarrow G / P
$$

and the associated line bundle

$$
\mathcal{L}_{Q} \rightarrow G / P \text {. }
$$

We have

$$
\operatorname{ch}_{2}(G / Q)=0
$$

Indeed, there is at least one $\mathrm{CDO}$ on $G / Q$ that can be defined via the chiral Hamiltonian reduction as follows. By analogy with Section 6.2, since (6.4) holds true with $S L_{2}$ replaced with an arbitrary simple complex Lie group $G$, we observe that if $\mathfrak{q}=\operatorname{Lie}(Q)$, then $H^{\infty / 2}\left(L \mathfrak{q}, \mathfrak{q} ; D_{G,(\cdot, \cdot)}^{\text {ch }}\right)$ is well defined for precisely one choice of $(\cdot, \cdot)$. In fact, in this case condition (6.2) amounts to the requirement that the restriction $(\cdot, \cdot)$ to $M_{1}$ be equal to the Killing form on $M_{1}$, and there is one and only one way to achieve that by appropriately rescaling $(\cdot, \cdot)$ - this is where assumption (6.10) is crucial. It is rather clear that $H^{\infty / 2}\left(L \mathfrak{q}, \mathfrak{q} ; D_{G,(\cdot,)}^{\text {ch }}\right)$ is a $\operatorname{CDO}$ on $G / Q$.

As a consequence, we obtain a vertex algebra morphism

$$
\mathcal{U}_{\text {alg }} \mathcal{V}(\mathfrak{g})_{(\cdot, \cdot)} \rightarrow H^{\infty / 2}\left(L \mathfrak{q}, \mathfrak{q} ; D_{G,(\cdot,)}^{\mathrm{ch}}\right)
$$

for a uniquely determined bilinear form $(\cdot, \cdot)$.

Assertion (6.11) is of course analogous to the fact that $\operatorname{ch}_{2}\left(S L_{2} / N\right)=0$, which was discussed in Section 6.2. Unlike the $s l_{2}$-case, however, we have obtained not a family but a single CDO on $G / Q$, and this precludes the definition of a CDO on $\mathcal{L}_{Q}$ via the chiral Hamiltonian reduction. It is then natural to expect that $\operatorname{ch}_{2}\left(\mathcal{L}_{Q}\right) \neq 0$ and that each CDO on $G / Q$ with $\mathcal{U}_{\text {alg }} \mathcal{V}(\mathfrak{g})_{(\cdot, \cdot)^{-}}$ structure is isomorphic to $H^{\infty / 2}\left(L \mathfrak{q}, \mathfrak{q} ; D_{G,(\cdot, \cdot)}^{\text {ch }}\right)$.

One example of this analysis is provided by $G=S L_{n}$ with $Q$ chosen to be the subgroup with 1st column equal to $(1,0,0, \ldots, 0)$. Then $G / Q$ is $\mathbb{C}^{n} \backslash 0$, the corresponding CDO has been used in Section 5.3, and it is easy to check that in this case the embedding (6.12) becomes precisely (5.24), (5.25).

Another example is provided by the space of pure spinors punctured at a point. It is a homogeneous space which satisfies assumption (6.10). Therefore, our analysis is an alternative 
way to prove the vanishing of the 2 nd component of the Chern character, originally verified by Nekrasov [24].

Needless to say, our discussion is very close in spirit to the definition of Wakimoto modules due to Wakimoto and Feigin-Frenkel, see [11] and references therein. In fact, it is easy to see that the spaces of sections over 'the big cell' of the sheaves constructed in Section 6.2 contain Wakimoto modules over $\widehat{s l}_{2}$, and those of the present section contain the so-called generalized Wakimoto modules corresponding to $R$.

\section{Acknowledgements}

The author would like to thank N. Nekrasov and especially V. Hinich for interesting discussions and for bringing [18] to his attention. The paper was completed at the IHES in Bures-sur-Yvette. We are grateful to the institute for hospitality and excellent working conditions. This work was partially supported by an NSF grant. Special thanks go to V. Gorbounov and V. Schechtman.

\section{References}

[1] Arkhipov S., Gaitsgory D., Differential operators on the loop group via chiral algebras, Int. Math. Res. Not. 2002 (2002), no. 4, 165-210, math.AG/0009007.

[2] Behrend K., Differential graded schemes I: Perfect resolving algebras, math.AG/0212225.

[3] Backelin J., Fröberg R., Koszul algebras, Veronese subrings and rings with linear resolutions, Rev. Roumaine Math. Pures Appl. 30 (1985), no. 2, 85-97.

[4] Berkovits N., Nekrasov N., The character of pure spinors, Lett. Math. Phys. 74 (2005), 75-109, hep-th/0503075.

[5] Berkovits N., Super-Poincaré covariant quantization of the superstring, J. High Energy Phys. 2000 (2000), no. 4, 18, 17 pages, hep-th/0001035.

[6] Bezrukavnikov R., Koszul property and Frobenius splitting of Schubert varieties, alg-geom/9502021.

[7] Bressler P., The first Pontryagin class, Compos. Math. 143 (2007), 1127-1163, math.AT/0509563.

[8] Courant T.J., Dirac manifolds, Trans. Amer. Math. Soc. 319 (1990), 631-661.

[9] Dorfman I., Dirac structures of integrable evolution equations, Phys. Lett. A 125 (1987), no. 5, 240-246.

[10] Frenkel E., Private communication.

[11] Frenkel E., Wakimoto modules, opers and the center at the critical level, Adv. Math. 195 (2005), 297-404, math.QA/0210029.

[12] Frenkel E., Ben-Zvi D., Vertex algebras and algebraic curves, 2nd ed., Mathematical Surveys and Monographs, Vol. 88, American Mathematical Society, Providence, RI, 2004.

[13] Feigin B., Semi-infinite homology of Lie, Kac-Moody and Virasoro algebras, Uspekhi Mat. Nauk 39 (1984), no. 2, 195-196 (in Russian).

[14] Feigin B., Parkhomenko S., Regular representation of affine Kac-Moody algebras, in Algebraic and geometric methods in mathematical physics (Kaciveli, 1993), Math. Phys. Stud., Vol. 19, Kluwer Acad. Publ., Dordrecht, 1996, 415-424, hep-th/9308065.

[15] Gorbounov V., Malikov F., Schechtman V., Gerbes of chiral differential operators. II. Vertex algebroids, Inv. Math. 155 (2004), 605-680, math.AG/0003170.

[16] Gorbounov V., Malikov F., Schechtman V., On chiral differential operators over homogeneous spaces, Int. J. Math. Math. Sci. 26 (2001), no. 2, 83-106, math.AG/0008154.

[17] Gorbounov V., Schechtman V., Homological algebra and divergent series, arXiv:0712.3670.

[18] Hinich V., Homological algebra of homotopy algebras, Comm. Algebra 25 (1997), 3291-3323, q-alg/9702015.

[19] Inamdar S.P., Mehta V.B., Frobenius splitting of Schubert varieties and linear syzygies, Amer. J. Math. 116 (1994), 1569-1586.

[20] Kac V., Vertex algebras for beginners, 2nd ed., University Lecture Series, Vol. 10, American Mathematical Society, Providence, RI, 1998. 
[21] Li H., Abelianizing vertex algebras, Comm. Math. Phys. 259 (2005), 391-411, math.QA/0409140.

[22] Liu Z.-J., Weinstein A., Xu P., Manin triples for Lie bialgebroids, J. Differential Geom. 45 (1997), 547-574, dg-ga/9508013.

[23] Malikov F., Lagrangian approach to sheaves of vertex algebras, Comm. Math. Phys. 278 (2008), 487-548, math.AG/0604093.

[24] Nekrasov N., Lectures on curved beta-gamma systems, pure spinors, and anomalies, hep-th/0511008.

[25] Primc M., Vertex algebras generated by Lie algebras, J. Pure Appl. Algebra 135 (1999), 253-293, math.QA/9901095. 